\title{
Analyse de la température de la Loire moyenne en été sur la période 1949 à 2003
}

\section{Analysis of the mid-Loire temperature in summer (1949-2003)}

\author{
Ph. Gosse ${ }^{(1)}$, J. Gailhard(2), F. Hendrickx(1) \\ (1) EDF R\&D, Département LNHE, 6 quai Watier, 78400 Chatou, France \\ (2) EDF DTG, 21 avenue de l'Europe, 38040 Grenoble, France \\ e-mail : philippe.gosse@edf.fr
}

\begin{abstract}
Résumé - On examine la capacité de deux modèles thermiques déterministes à base physique (CALNAT et COURNAT) à reproduire l'augmentation de la température moyenne estivale de la Loire à Dampierre-en-Burly sur la période 1980-2003. Ces modèles utilisent en entrée les chroniques de quatre paramètres atmosphériques relevés à pas tri-horaire à la station Météo-France de Tours. CALNAT calcule la température naturelle locale d'un plan d'eau sans considération du débit fluvial, tandis que le code dérivé COURNAT propage une masse d'eau dans les conditions de débit mesurées à la station de Gien. En été, du 15 juin au 15 septembre, l'échauffement moyen sur les 24 ans observé à la station de mesures en continu des eaux de la Loire située à l'amont de la centrale nucléaire de Dampierre-en-Burly atteint $1,85^{\circ} \mathrm{C}$. Une tendance comparable $\left(2,0^{\circ} \mathrm{C}\right)$ est observée à la station de Belleville amont située une trentaine de kilomètres en amont. Les échauffements moyens calculés sur la même période par CALNAT et COURNAT sont respectivement de $1,55^{\circ} \mathrm{C}$ et $1,7^{\circ} \mathrm{C}$; ils représentent ainsi $84 \%$ et $92 \%$ de la tendance d'échauffement découlant des mesures à Dampierre-en-Burly. Durant cette période d'étiage, l'essentiel de l'échauffement de la Loire est attribuable aux variations tendancielles de la température d'air $\left(+1,5^{\circ} \mathrm{C}\right)$, de la pression de vapeur d'eau $(+0,65 \mathrm{hPa})$, de la vitesse de vent à 10 mètres $(-0,4 \mathrm{~m} / \mathrm{s})$, et de la nébulosité du ciel $(-0,15$ octa) dont la contribution à l'élévation de la température naturelle locale peut être évaluée à l'aide de CALNAT à respectivement $46 \%, 18 \%, 28 \%$ et $8 \%$. L'application de CALNAT et COURNAT sur la période 1949-2003 donne un échauffement moyen tendanciel de l'eau de l'ordre de $0,5^{\circ} \mathrm{C}$ sur 55 ans pour le même trimestre 15 juin- 15 septembre. Le débit ne présentant pas de tendance d'évolution significative sur la période, cet échauffement modéré comparativement à celui de 1980-2003 est attribuable au faible effet global des tendances de variation des quatre paramètres atmosphériques. Contrairement à la période 1980-2003, les quatre grandeurs atmosphériques présentent des variations à effets antagonistes sur la température d'eau de 1949 à 2003 : réparties uniformément à chaque pas tri-horaire de calcul, les évolutions estimées de la température d'air $\left(+0,9^{\circ} \mathrm{C}\right)$, de la pression de vapeur d'eau $(-0,55 \mathrm{hPa})$, de la vitesse du vent à $10 \mathrm{~m}(+0,4 \mathrm{~m} / \mathrm{s})$ et de la nébulosité $(-0,4$ octa) sur ces 55 ans à l'échelle trimestrielle modifient la température naturelle locale de l'ordre de $+0,4^{\circ} \mathrm{C},-0,2^{\circ} \mathrm{C},-0,4^{\circ} \mathrm{C}$ et $+0,3^{\circ} \mathrm{C}$ respectivement. Ces résultats suggèrent qu'une
\end{abstract}


relation linéaire observée entre variations de températures d'air et de plan d'eau n'est pas systématiquement extrapolable sur un plus grand nombre de décennies. Comme ceux de COURNAT, les résultats de CALNAT indiquent que, sur la période 1949-2003, 2003 est l'année record pour la dose thermique maximale reçue par l'écosystème fluvial aussi bien sur 7 jours consécutifs que sur 90 jours consécutifs (avec des températures moyennes calculées de respectivement $29,5^{\circ} \mathrm{C}$ et $25^{\circ} \mathrm{C}$ confirmées par la station de mesure de Dampierre). Pour la dose thermique maximale reçue sur 30 jours consécutifs, CALNAT place 2003 dans les années de tête avec une température moyenne de $26{ }^{\circ} \mathrm{C}$, confirmée par la station de Dampierre. Ces pics thermiques de 2003 remarquablement reproduits à $0,3^{\circ} \mathrm{C}$ près par CALNAT aux échelles de temps considérées sont ainsi expliqués par les conditions atmosphériques locales exceptionnelles et non par le très bas débit fluvial (minimum de 50 m³/s à Gien) alors observé. À l'opposé, l'été 1977 ressort avec COURNAT plus adapté lorsque les débits sont élevés - comme l'été le plus froid de la période 19492003 pour la dose thermique maximale reçue sur 7,30 et 90 jours avec des températures moyennes de respectivement $22^{\circ} \mathrm{C}, 20^{\circ} \mathrm{C}$ et $19^{\circ} \mathrm{C}$, effectivement observées. Pour la dose thermique maximale reçue par l'écosystème Loire sur 30 jours, les années 1976 et 1977 se distinguent par la plus forte différence entre deux années consécutives sur la période 1949-2003.

Mots clés - température, Loire, rivière, modèle, changement climatique

Abstract - We examine the ability of two physics-based deterministic thermal models (CALNAT and COURNAT) to reproduce the increase of the Loire river temperature observed in summer at Dampierre-en-Burly over the 1980-2003 periods. These models use input data sets of four atmospheric parameters, collected every three hours, at the Météo-France station of Tours. CALNAT calculates the local natural temperature of a water body without taking into account the river discharge, whereas COURNAT, a derivate code, propagates a water body under the Gien discharges conditions. In summer, trimester 15 June-15 September, the average warming observed from 1980 to 2003 in the Loire, at the thermal recorders installed upstream of Dampierre-en-Burly nuclear power plant, reached $1.85^{\circ} \mathrm{C}$. A comparable increase $\left(2.0^{\circ} \mathrm{C}\right)$ is obtained about thirty kilometers upstream, at the station upstream of the Belleville nuclear power plant. The average warming trends calculated over the 24 years by CALNAT and COURNAT are respectively $1.55^{\circ} \mathrm{C}$ and $1.7^{\circ} \mathrm{C}$; they represent $84 \%$ and $92 \%$ of the warming trend resulting from the measurements at Dampierre-en-Burly. During this low flow period, most of the warming trend of the Loire can be linked to air temperature $\left(+1.5^{\circ} \mathrm{C}\right)$, water vapor pressure $(+0.65 \mathrm{hPa})$, wind velocity at $10 \mathrm{~m}(-0.4 \mathrm{~m} / \mathrm{s})$ and sky nebulosity $(-0.15$ octa) variation trends, whose contribution to the local natural temperature can be estimated at $46 \%, 18 \%, 28 \%$ and $8 \%$ respectively. The application of CALNAT and COURNAT to the 1949-2003 periods gives a warming trend of water close to $0.5^{\circ} \mathrm{C}$ over 55 years for the same trimester 15 June-15 September. Without any significant variation trend detected for the river discharge, this rather moderate warming compared to the 1980-2003 period can be linked to the antagonistic effect of the 4 atmospheric parameters variation trends: applied uniformly every three hourly calculation step, over 55 years, the quarterly trends of air temperature $\left(+0.9^{\circ} \mathrm{C}\right)$, water vapor pressure $(-0.55 \mathrm{hPa})$, wind velocity at $10 \mathrm{~m}(+0.4 \mathrm{~m} / \mathrm{s})$ and sky nebulosity $(-0.4$ octa) modify the local natural temperature by around $+0.4^{\circ} \mathrm{C},-0.2^{\circ} \mathrm{C},-0.4^{\circ} \mathrm{C}$ et $+0.3^{\circ} \mathrm{C}$ respectively. These results question the pertinence of extrapolating over more decades a linear relationship found between air and water body temperatures. Like COURNAT, CALNAT results indicate that 2003 is an exceptional year of the 1949-2003 periods, for the maximal thermal dose received by the river ecosystem during 7 and 90 consecutive days, with average water temperatures of $29.5^{\circ} \mathrm{C}$ and $25^{\circ} \mathrm{C}$ confirmed by the Dampierre measurement station. For the maximal thermal dose received during 30 consecutive days, year 2003 ranks within 
the top years with an average temperature of $26^{\circ} \mathrm{C}$. It must be noted that these thermal peaks of 2003 which are remarkably reproduced (with a $0.3^{\circ} \mathrm{C}$ accuracy) by CALNAT are explained by the extreme values of the 4 atmospheric parameters and not by the very low river discharge observed at that time (minimum of $50 \mathrm{~m}^{3} / \mathrm{s}$ at Gien). On the opposite, summer 1977 is disclosed by COURNAT - more adapted than CALNAT to high discharges as the coldest summer of the 1949-2003 period for the maximal thermal dose received during 7,30 and 90 consecutive days with an average temperature - confirmed by the measurements - close to $22^{\circ} \mathrm{C}, 20^{\circ} \mathrm{C}$ and $19{ }^{\circ} \mathrm{C}$ respectively. As regards the maximal thermal dose received during 30 consecutive days, 1976 and 1977 show by far the highest difference between two consecutive years.

Key words - temperature, Loire, river, model, climate change

\section{INTRODUCTION}

La température naturelle locale ( $\left.T_{\text {nat }}\right)$ peut être définie comme la température prise par un plan d'eau homogène sans entrée d'eau ni échange significatif d'énergie avec le fond. L'évolution au cours du temps de $\mathrm{T}_{\text {nat }}$ ne dépend alors que des échanges d'énergie à l'interface eau-atmosphère et donc des conditions météorologiques locales et de la profondeur du plan d'eau. On dit aussi que le plan d'eau est à l'équilibre avec les conditions météorologiques locales. Dans son cheminement vers l'aval, une rivière a une température qui tend en permanence vers la température naturelle locale correspondant à sa profondeur. La rivière ne peut atteindre la température naturelle locale qu'à partir d'une certaine distance des sources ou en s'éloignant des discontinuités thermiques marquées (grand aménagement hydraulique, affluent à histoire hydroclimatologique différente, résurgence de nappe,...), cette distance étant dépendante de la vitesse de l'écoulement (Gras, 1969, 1970; Gras et al., 1983, 1986 ; Jacquet, 1983).

Développé à EDF dans les années 1970, CALNAT - dont les équations sont précisées en annexe - est un modèle déterministe à base physique (c'est-à-dire mettant en équation les échanges d'énergie) qui calcule $T_{\text {nat }}$ à pas tri-horaire en exploitant les observations tri-horaires de température d'air, d'humidité de l'air, de nébulosité du ciel, de vitesse du vent et de pression atmosphérique acquises à une station météorologique voisine. II a été montré que CALNAT permettait de reconstituer sur plusieurs mois d'affilée avec une précision meilleure que le degré (hors période de crue) des chroniques de températures journalières des parties moyennes ou aval de rivières comme la Loire, la Marne, la Moselle, l'Oise, la Saône ou la Seine (Gras et al., 1971, 1986). La température d'une rivière peut cependant n'être égale à $T_{\text {nat }}$ que pendant une courte période de l'année, comme par exemple la Garonne près du confluent du Tarn (Gosse et Gailhard, 1996) ou même qu'exceptionnellement si elle subit en permanence l'influence de grands lacs amont ou si son écoulement rapide lui fait franchir en peu de temps des zones à climats différents, comme c'est le cas du Rhône ou du Rhin (Poulin, 1980 ; Gras et al., 1986).

De nombreuses études réalisées à EDF R\&D (Gosse, 2005; Dupeyrat et Bertier, 2006 ; pour les plus récentes) 
ont confirmé que la Loire moyenne était à la température naturelle locale une grande partie de l'année, en particulier l'été : l'application de CALNAT avec les données météorologiques de la station Météo-France de Tours permet en effet de reconstituer les températures journalières de cette partie du fleuve avec une précision de l'ordre du degré Celsius.

L'objet de cet article est tout d'abord de préciser la tendance à l'échauffement de la Loire moyenne en été sur la période 1949-2003 à partir de calculs thermiques effectués avec CALNAT et avec un code dérivé COURNAT qui prend en compte l'effet refroidissant des hauts débits en été, et de comparer ces résultats avec les échauffements constatés depuis 1980 en Loire aux stations de mesures en continu de la température du fleuve situées dans le proche amont de la prise d'eau de la centrale nucléaire de Dampierreen-Burly (dite par la suite station Dampierre amont). 1949 est la première année de simulation car elle correspond au début des enregistrements, à la station météorologique de Tours, de l'ensemble des paramètres atmosphériques considérés par CALNAT. II se trouve que 1949 est aussi l'année d'observation du plus sévère étiage de la Loire sur la période étudiée, une situation de même gravité n'étant pas apparue en 2003 grâce au soutien d'étiage du fleuve via les retenues de Naussac sur l'Allier et Villerest sur la Loire mises en service en 1983 et 1984 (Moreau, 2004). Cela nous conduira à évaluer la pertinence de CALNAT et COURNAT relativement aux étiages sévères.

Outre l'examen des capacités descriptives de CALNAT et de COURNAT, nous préciserons les informations supplémentaires que peut apporter notre approche déterministe à base physique par rapport à une approche purement statistique comme celle de Moatar et Gailhard (2006) que nous reprendrons. Leur modèle de régression linéaire multiple permet d'estimer la température moyenne trimestrielle de la Loire moyenne à partir de celle de l'air et du débit du fleuve, relation qu'ils ont utilisée pour constituer par extrapolation une chronique de températures moyennes de la Loire moyenne de 1881 à 2003 sur le trimestre juin-août.

\section{LES MODĖLES ET LES DONNÉES UTILISÉS}

\subsection{Les chroniques de mesures de température d'eau de Loire}

Des chroniques de température mesurées en Loire moyenne à pas horaire sont disponibles sur la période 1980-2003 à Belleville (situé près de $30 \mathrm{~km}$ en amont de Dampierre), Dampierre, Saint-Laurent-des-Eaux et Avoine. Ces deux dernières stations, situées en aval de Dampierre et d'Orléans, correspondent au proche amont des centrales nucléaires de Saint-Laurent-des-Eaux et Chinon respectivement. Dans cet article, nous privilégierons les stations de Belleville et de Dampierre. En effet, Moatar et Gailhard (2006) ont mis en évidence que la température à la station de Saint-Laurent était sensible aux apports de la nappe; et celle d'Avoine est également influencée, notamment par les apports du Cher.

La station de Dampierre présente le double intérêt d'être plus proche que Belleville de l'aire climatique de 
Tours et moins influencée thermiquement par les masses d'eau du bassin versant amont. On considère deux séries de mesure à cette station sur la période 1980-2003 dont les résultats exploités sont les moyennes journalières des températures enregistrées:

- la première est constituée à partir des données du capteur EDF/DTG placé en Loire à l'amont de la centrale de Dampierre que nous appellerons « Dampierre amont série 1 » ; cette série a été exploitée par Moatar et Gailhard (2006);

- la deuxième que nous appellerons « Dampierre amont - série 2 » est constituée à l'aide du capteur DTG Dampierre amont, et des stations multi-paramètres (température oxygène dissous - $\mathrm{pH}$ - conductivité) situées à l'amont et à l'aval (Ouzouer-sur-Loire) de la centrale de Dampierre. La considération de cette deuxième série est un moyen d'évaluer la variabilité des mesures de température d'eau et l'effet de cette variabilité sur la tendance estimée de l'échauffement de la Loire. On exploite la totalité du fichier informatique disponible des données du capteur de la station multi-paramètres amont (années 1993-2003) : pour ces 11 ans, on remplace les données de la série 1 de Dampierre amont par celles de la station multi-paramètres, si l'écart amont-aval des deux stations multi-paramètres ne dépasse pas $1^{\circ} \mathrm{C}$. Cette deuxième série de données reprend aussi pour la restitution de la moyenne trimestrielle 15 juin - 15 septembre les données du capteur de la station multiparamètres amont pour l'année
1991(1), compte tenu de l'écart moyen important relevé avec le capteur DTG pour cette année là. Pour les autres années de la période 1980-2003, les données de la série 2 sont les mêmes que celles de la série 1 Dampierre amont.

Les moyennes trimestrielles de ces deux séries de mesures sont présentées sur la figure 5 .

\subsection{Description des modèles et paramètres de calage}

On applique le code CALNAT dont les équations sont données en annexe en prenant comme entrée les chroniques tri-horaires des quatre paramètres atmosphériques - température d'air et pression de vapeur d'eau entre 1 et 2 mètres du sol, vitesse du vent à 10 mètres (moyenne sur 10 minutes) et nébulosité du ciel - relevés par Météo-France à la station de Tours, la pression atmosphérique qui a très peu d'influence sur les résultats étant prise constante et égale à la valeur moyenne de la station. II y a quatre paramètres de calage possibles dans le code CALNAT :

- la profondeur d'inertie thermique $\mathrm{H}$ qui est prise plus ou moins proche de la profondeur moyenne de l'écoulement en fonction de la réponse du modèle aux échelles de temps considérées pour l'analyse, sachant que son choix est plus déterminant pour la représentation du cycle thermique diurne d'une rivière que pour l'évaluation d'une moyenne mensuelle

(1) Données extraites du rapport de surveillance 1991 de la centrale de Dampierre. 
de température. II est toutefois possible de calculer $\mathrm{H}$ à partir d'une courbe en puissance du débit comme dans COURNAT;

- les coefficients $a$ et $b$ présents dans la fonction de vent $(\mathrm{a}+\mathrm{bV})$ - avec V vitesse du vent $(\mathrm{m} / \mathrm{s})$ à 10 mètres du sol - qui définit le rôle du vent dans les flux d'échange d'énergie par évaporation (CE) et convection (CV) à l'interface eau-atmosphère ;

- l'émissivité de l'air eair (adimensionnel) qui apparaît dans la formulation du rayonnement atmosphérique RA et devrait ne pas être trop éloignée de la formule théorique qui est : $9.10^{-6} \times\left(T_{\text {air }}+273,15\right)^{2}$, avec $\mathrm{T}_{\text {air }}$ température d'air en ${ }^{\circ} \mathrm{C}$.

Pour l'application du modèle CALNAT, la profondeur d'inertie thermique est prise constante et égale à 2 mètres; $a, b$ et $e_{\text {air }}$ sont respectivement égaux à $0,002,0,0012^{(2)}$ et 0,8 .

(2) R. Gras - « père » de CALNAT - a considéré que l'équation $b=0,6 a$ pouvait être adoptée d'une part parce qu'il a été évalué qu'une fonction de vent à un seul paramètre de calage (a) et de la forme $a\left(1+V_{2}\right)$ avec $V_{2}$ vitesse de vent mesurée à $2 \mathrm{~m}$ du sol en $\mathrm{m} / \mathrm{s}$ est suffisante dans le cas général (Salençon et Thébault, 1997) et d'autre part parce que la vitesse de vent à 2 mètres du sol est proche de $60 \%$ de la vitesse de vent à $10 \mathrm{~m}$ du sol. Cette formulation simplifiée de la fonction de vent revient à dire que même pour un vent mesuré nul à une station météo proche, il règne sur la rivière un vent résiduel de l'ordre de $1 \mathrm{~m} / \mathrm{s}$ induit par les différences de température entre le plan d'eau et le sol, le relief dans la vallée ou la présence d'arbres, les situations de convection libre pure étant rares. La pertinence de l'équation suppose toutefois que la vitesse moyenne du vent mesurée à la station météorologique considérée comme représentative est proche de celle observable sur le plan d'eau étudié.
L'expérience a montré que ces trois dernières valeurs - dites standard sont généralement proches de l'optimum dans les applications de CALNAT à divers sites et elles ont été de ce fait retenues dans un essai de régionalisation de la température naturelle locale des plans d'eau français (Gosse et al., 1997). Gosse (2005) a par ailleurs montré que ce jeu de paramètres permettait de reproduire avec une précision meilleure que le degré Celsius les courbes classées de températures journalières de la Loire issues des enregistrements de la station de mesure de Dampierre amont sur la période 1980-2003 (série 1).

Une version dérivée de CALNAT est également testée. II s'agit du code COURNAT utilisé par EDF comme outil d'aide à la prévision à 9 jours d'échéance de la température de la Loire moyenne sur la base de prévisions météorologiques fournies par Météo-France pour la station de Tours et de prévisions hydrologiques. COURNAT reprend les équations d'échange d'énergie à l'interface eau-atmosphère de CALNAT mais il propage $^{(3)}$ une masse d'eau à une vitesse $U$ sur un tronçon de longueur théorique $\mathrm{D}$ depuis une source fictive de température $T_{\text {source }}$ dans un écoulement ayant une profondeur d'inertie thermique $\mathrm{H}$ fonction du débit. $\mathrm{U}(\mathrm{m} / \mathrm{s})$ est déterminé par l'équation $U=Q^{m_{+}}$ $\mathrm{n}$, avec $\mathrm{Q}$ débit à Gien en $\mathrm{m}^{3} / \mathrm{s}$ et $\mathrm{I}$, $m$ et $n$ constantes calées dépendant du choix de $D$ pris égal à $230 \mathrm{~km}$.

(3) COURNAT utilise le module de transport non permanent du code MOTRICE (Gilbert et Roult, 1986). 
$H(m)$ est déterminé par l'équation $\mathrm{H}=\mathrm{c} \times \mathrm{Q}^{\mathrm{d}}$, avec $\mathrm{Q}$ débit $\left(\mathrm{m}^{3} / \mathrm{s}\right)$ à Gien et $c$ et $d$ constantes calées. $\mathrm{T}_{\text {source }}\left({ }^{\circ} \mathrm{C}\right)$, par analogie au comportement d'une eau de nappe, a une évolution annuelle sinusoïdale déterminée par l'équation $\mathrm{T}_{\text {source }}=\mathrm{T}_{0}+$ $\mathrm{A} / 2 \times \cos \left(2 \pi \times\left(\left(\mathrm{J}-\mathrm{J}_{0}\right) / 365+0,5\right)\right)$, avec $\mathrm{J}$ jour de l'année calendaire et $\mathrm{T}_{0}$, $\mathrm{A}$ et $\mathrm{J}_{0}$ constantes à caler. Le modèle COURNAT permet de prendre en compte les phénomènes suivants :

- une masse d'eau dans son écoulement vers l'aval tend vers la température naturelle locale correspondant à sa profondeur ;

- au-dessus d'un certain débit, la masse d'eau est soumise aux influences hydro-climatiques amont.

Dans son application à cette étude, COURNAT a les mêmes entrées que CALNAT - données atmosphériques de Tours - auxquelles s'ajoute la chronique de débits journaliers à Gien. Les paramètres de COURNAT ont été déterminés sur la période 1991-2003 par calage automatique sur les données journalières du capteur DTG mesurant en continu la température de la Loire à Belleville. Le calage automatique a été effectué en utilisant une méthode d'algorithme génétique avec le critère de Nash comme contrôle. Les valeurs obtenues pour les paramètres du modèle sont les suivantes: $c=0,161 ; d=0,453 ; \quad I=0,486$; $m=0,129 ; n=-0,624 ; T_{0}=9,084 ;$ $A=11,364 ; J_{0}=27 ; e_{\text {air }}=0,819$; $a=0,00488 ; b=0,00036$.

Le calage automatique utilisé pour COURNAT conduit à la sélection d'un paramètre a près de deux fois plus élevé et d'un paramètre $b$ près de 5 fois plus faible que ceux de CALNAT. Cela donne une fonction de vent $\mathrm{a}+\mathrm{bV}$ moins variable, avec diminution du poids de la donnée de vent. Les deux modèles donnent la même valeur à $1 \%$ près de la fonction de vent pour un vent de $3,5 \mathrm{~m} / \mathrm{s}$ à 10 mètres, qui est la vitesse moyenne en été à la station de Tours sur la période 1980-2003. De même, le calage automatique sélectionne une valeur $e_{\text {air }}$ s'écartant de moins de $3 \%$ de celle de CALNAT.

On notera que le lancement de CALNAT et de COURNAT nécessite l'introduction d'une condition initiale de température d'eau, mais cette valeur n'a pas d'influence sur les résultats au bout de quelques jours dans la gamme de profondeur considérée.

Nous testerons également une approche de modélisation purement statistique (régression linéaire multiple) consistant à reconstituer chaque année la température de la Loire en moyenne sur les trimestres juin-août et 15 juin-15 septembre à l'aide d'une équation multilinéaire retenant comme variables explicatives la moyenne trimestrielle des températures d'air à Orléans ou Tours et le logarithme népérien de la moyenne trimestrielle des débits du fleuve à Gien. C'est l'approche utilisée par Moatar et Gailhard (2006) pour constituer une chronique de températures moyennes de la Loire sur la période juin-août de 1881 à 2003. Nous développerons trois versions de ce modèle statistique :

- une version dite «STAT1 » calée sur les moyennes trimestrielles de température d'eau (1 ${ }^{\text {er }}$ juin31 août) obtenues sur la période 1976-2003 à partir des données des quatre stations de mesure en continu de Belleville, Dampierre (série 1), Saint-Laurent et Avoine situées en amont des quatre centrales nucléaires de la Loire. Les données d'entrée de STAT1 
sont la température trimestrielle de l'air à Orléans ( $\mathrm{T}_{\text {air Orléans }}$ en ${ }^{\circ} \mathrm{C}$ ) et le logarithme népérien du débit moyen trimestriel à Gien $\left(Q_{\text {Gien }}\right.$ en $\mathrm{m}^{3} / \mathrm{s}$ ). II s'agit à quelques nuances près $^{(4)}$ du modèle construit par Moatar et Gailhard (2006). L'équation de régression trouvée applicable sur le trimestre $1^{\mathrm{er}}$ juin -31 août est :

$$
\begin{gathered}
\mathrm{T}_{\text {Loire }}\left({ }^{\circ} \mathrm{C}\right)=11,95+0,74 \mathrm{~T}_{\text {air Orléans }} \\
-0,83 \mathrm{Ln} \mathrm{Q}_{\text {Gien }} ;
\end{gathered}
$$

- une version «STAT2 » calée sur les moyennes trimestrielles ( $1^{\mathrm{er}}$ juin31 août) de température d'eau à la station de Dampierre (série 1) pour la période 1980-2003. Les données d'entrée de STAT2 sont la température trimestrielle de l'air à Tours $\left(\mathrm{T}_{\text {air Tours }}\right.$ en ${ }^{\circ} \mathrm{C}$ ) et le logarithme népérien du débit moyen trimestriel à Gien ( $Q_{\text {Gien }}$ en $\left.\mathrm{m}^{3} / \mathrm{s}\right)$. L'équation de régression trouvée applicable sur le trimestre $1^{\mathrm{er}}$ juin31 août est :

$$
\begin{aligned}
\mathrm{T}_{\text {Loire }}\left({ }^{\circ} \mathrm{C}\right)= & 15,84+0,63 \mathrm{~T}_{\text {air Tours }} \\
& -1,13 \mathrm{Ln} \mathrm{Q}_{\text {Gien }} ;
\end{aligned}
$$

- une version «STAT3 » calée sur les moyennes trimestrielles (15 juin-15 septembre) de température d'eau à la station de Dampierre (série 1) pour la période 1980-2003. Les données d'entrée sont également la température trimestrielle de l'air

(4) Moatar et Gailhard (2006) ont utilisé le débit de la Loire à Blois et les mesures de température de deux stations (Dampierre et Avoine). Le modèle STAT1 présente sur la période 19762003 un écart-type des écarts calcul-mesure de $0,36^{\circ} \mathrm{C}$, qui est très proche de celui donné par Moatar et Gailhard $\left(0,3^{\circ} \mathrm{C}\right)$. à Tours ( $\mathrm{T}_{\text {air Tours }}$ en ${ }^{\circ} \mathrm{C}$ ) et le logarithme népérien du débit moyen trimestriel à Gien $\left(Q_{\text {Gien }}\right.$ en $\left.\mathrm{m}^{3} / \mathrm{s}\right)$. L'équation de régression trouvée applicable sur le trimestre 15 juin15 septembre est :

$$
\begin{aligned}
\mathrm{T}_{\text {Loire }}= & 16,42+0,56 \mathrm{~T}_{\text {air }} \text { Tours } \\
& -1,03 \mathrm{Ln} \mathrm{Q}_{\text {Gien }} .
\end{aligned}
$$

La capacité des modèles CALNAT, COURNAT, STAT2 et STAT3 à reproduire les températures moyennes trimestrielles de la Loire à Dampierre sur la période 1980-2003 est examinée dans le tableau I qui donne la moyenne et l'écart-type des écarts calculs-mesures trouvés chaque année. On constate que tous ces modèles présentent une qualité descriptive assez proche. Celle des modèles statistiques qui sont calés sur les mesures de températures utilisées pour la constitution du modèle apparaît un peu meilleure que celle de COURNAT calé sur les températures de Loire à Belleville et de CALNAT qui n'a pas été calé.

On note que les séries 1 et 2 Dampierre amont donnent le même niveau d'écart calculs-mesures avec CALNAT ou COURNAT. Mais la série 2 réduit les plus grands écarts calculs-mesures (années 1993, 1996 et 1997) observés avec la série 1 sur la période 1993-2003 (Fig. 1); elle réduit aussi en 1991 l'écart calculsmesures observé entre CALNAT et la série 1 (réduction de l'écart de $1^{\circ} \mathrm{C}$ à $0,2^{\circ} \mathrm{C}$ ) mais n'affecte pas celui relatif à COURNAT qui reste compris entre 0,6 et $0,7^{\circ} \mathrm{C}$. 
Tableau I. Moyenne et écart-type des écarts calcul-mesures trouvés chaque année de la période 1980-2003 entre les moyennes trimestrielles de températures d'eau observées au site Dampierre amont (série 1 ) et celles calculées par quatre modèles : CALNAT, COURNAT, STAT2 ( ${ }^{\text {er }}$ juin31 août) et STAT3 (15 juin-15 septembre).

Table I. Average and standard error of the calculation-measurements differences, found each year of the 1980-2003 period, between the quarterly averages of water temperatures observed close upstream of the Dampierre nuclear power plant (series 1) and those calculated by four models: CALNAT, COURNAT, STAT2 (1st June-31 August) and STAT3 (15 June-15 September).

\begin{tabular}{|l|c|c|c|c|}
\cline { 2 - 5 } \multicolumn{1}{c|}{} & \multicolumn{2}{c|}{1 juin - 31 août } & \multicolumn{2}{c|}{15 juin - 15 septembre } \\
\cline { 2 - 5 } \multicolumn{1}{c|}{} & moyenne & écart -type & moyenne & écart -type \\
\cline { 2 - 5 } \multicolumn{1}{c|}{} & ${ }^{\circ} \mathrm{C}$ & ${ }^{\circ} \mathrm{C}$ & ${ }^{\circ} \mathrm{C}$ & ${ }^{\circ} \mathrm{C}$ \\
\hline CALNAT & 0,05 & 0,6 & 0,1 & 0,5 \\
\hline COURNAT & 0,2 & 0,4 & 0,2 & 0,4 \\
\hline STAT2/STAT3 & 0 & 0,4 & 0 & 0,4 \\
\hline
\end{tabular}

\section{RÉSULTATS}

On génère avec CALNAT et COURNAT les températures tri-horaires d'eau sur la période 1949-2003. Les résultats exploités ici sont :

- les températures moyennes trimestrielles en été (trimestres 15 juin-15 septembre et $1^{\text {er }}$ juin31 août) pour les périodes 19802003 et 1949-2003;

- la moyenne de températures la plus élevée de l'année sur 7, 30 ou 90 jours consécutifs sur la période 1949-2003.

\subsection{Analyse de tendance des températures estivales calculées sur la période 1980-2003}

\subsubsection{5 juin-15 septembre}

Le trimestre 15 juin- 15 septembre a été choisi parce qu'il présente un bon compromis entre la période d'étiage (Fig. 2) et la période chaude de la Loire.

La figure 3 compare pour chaque année les températures trimestrielles calculées par CALNAT et COURNAT avec celles des données de Dampierre amont (série 2) et Belleville amont. Comme l'illustre la figure 4 , on constate que COURNAT est meilleur que CALNAT pour décrire les épisodes de crue estivale (au-delà de $400 \mathrm{~m}^{3} / \mathrm{s}$ ) pour lesquels la Loire moyenne n'est pas à la température naturelle locale. Cependant, en dehors des années 1981, 1987 et 1992, la Loire moyenne n'a pas connu de crue significative du 15 juin au 15 septembre entre 1980 et 2003, ce qui explique le bon accord d'ensemble entre COURNAT et CALNAT pour cette période de l'année.

Les accroissements des températures trimestrielles d'eau calculés et mesurés sur les 24 ans sont obtenus en utilisant un test de régression linéaire précisé dans l'annexe 2. Les résultats, leur significativité et l'intervalle de confiance à $90 \%$ de l'estimation de la tendance sont repris en figure 5 . La même démarche a été conduite pour les quatre paramètres atmosphériques température d'air, pression de vapeur d'eau, vitesse de vent et nébulosité relevés à la station météorologique de Tours ainsi que pour le débit de la Loire à Gien (Fig. 6). 


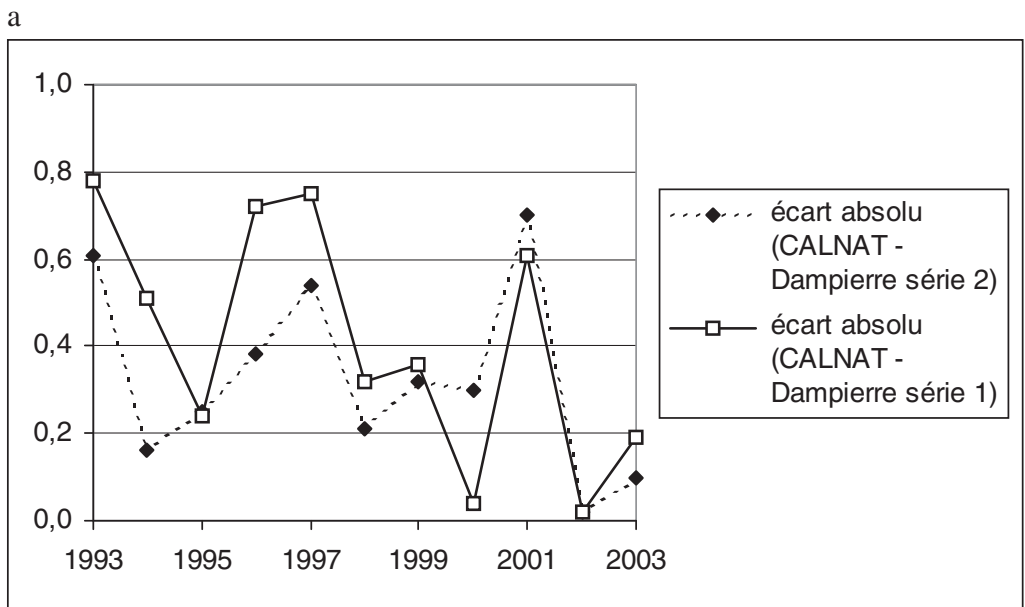

$\mathrm{b}$

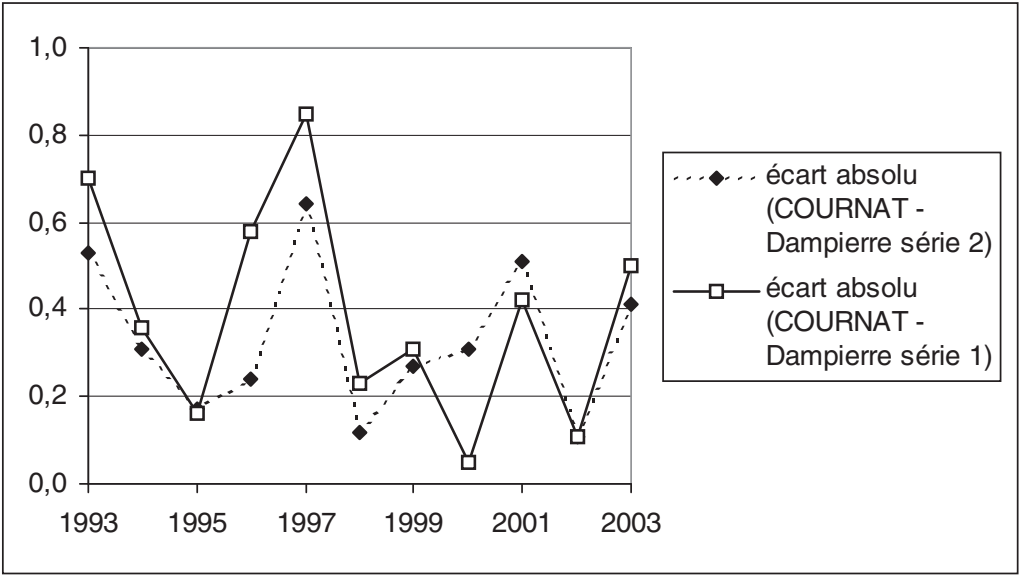

Fig. 1. Écart absolu entre les moyennes trimestrielles de température d'eau calculées par CALNAT et COURNAT et celles données par les séries 1 et 2 de mesures de températures journalières de la Loire dans le proche amont de la centrale de Dampierre (période 1993-2003).

Fig. 1. Absolute difference between quarterly averages of water temperatures calculated by CALNAT and COURNAT and those given by two measurement series (1 and 2) of Loire daily temperatures close upstream of Dampierre nuclear power plant (years 1993-2003).

Pour l'ensemble des paramètres analysés, la tendance sur 24 ans s'avère à chaque fois significative à plus de $97 \%$ (i.e. risque d'erreur de $3 \%$ en acceptant l'hypothèse d'une tendance différente de zéro) sauf pour la pression de vapeur d'eau et la nébulosité. Les tendances d'évolution trouvées 
Loire à Gien : Quantiles des Débits moyens journaliers (1949-2003)

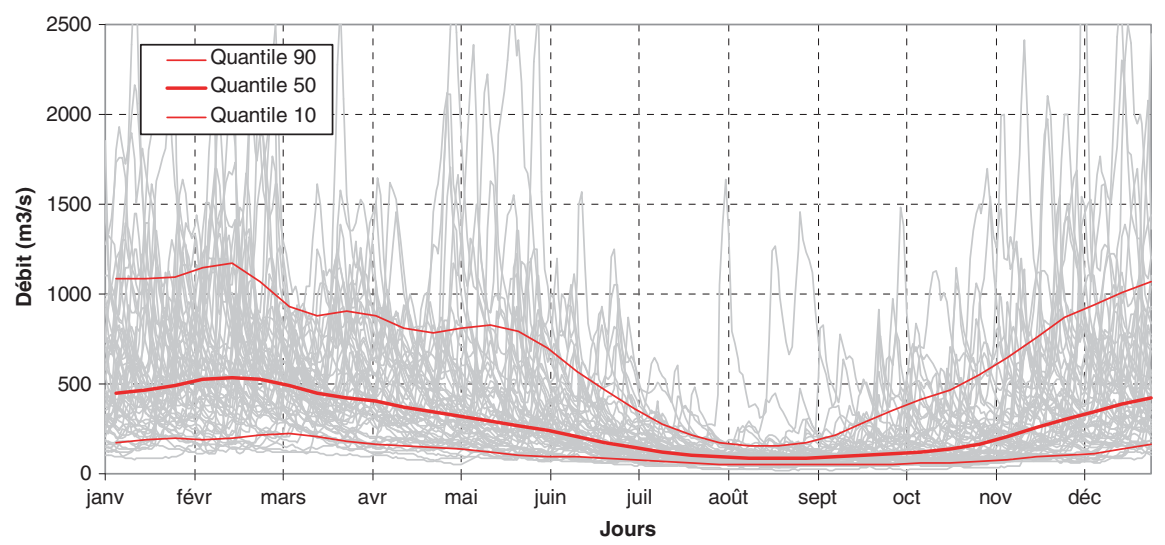

Fig. 2. Caractérisation des débits de la Loire moyenne à Gien (années 1949-2003).

Fig. 2. Characterisation of the mid-Loire discharges at Gien (years 1949-2003).

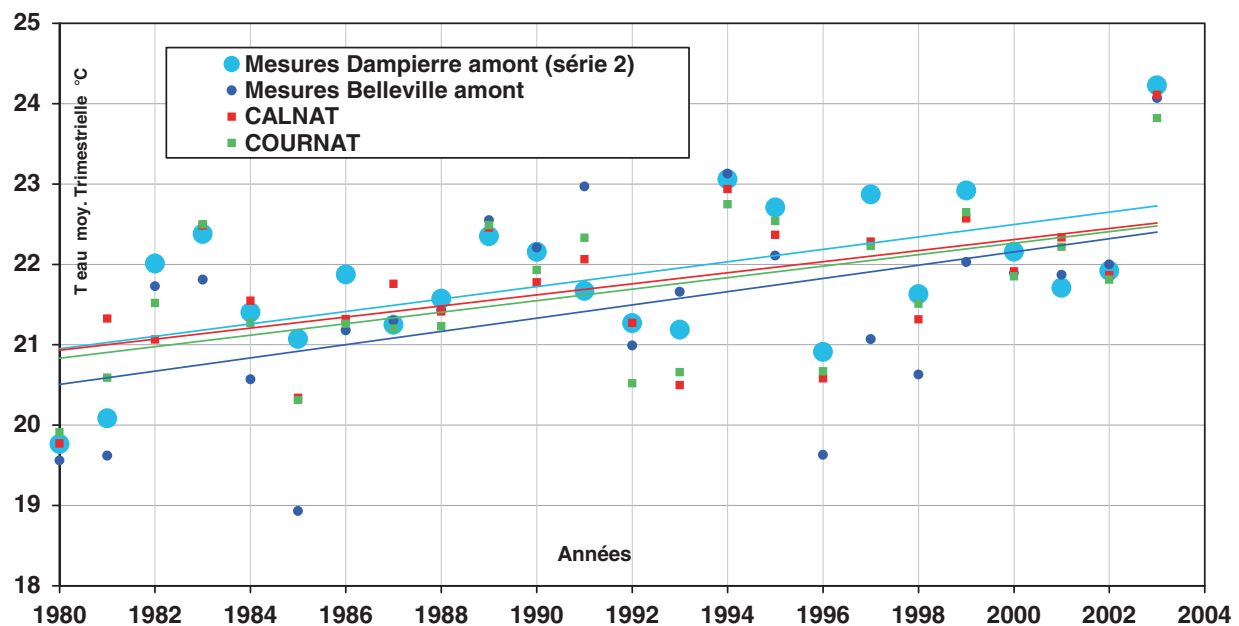

Fig. 3. Comparaison sur le trimestre 15 juin-15 septembre des moyennes des températures de Loire mesurées en continu à la station Dampierre amont (série 2), des températures d'air mesurées au pas tri-horaire à la station Météo-France de Tours et des températures d'eau calculées au pas trihoraire par CALNAT et COURNAT (période 1980-2003). Les courbes de la figure sont les droites de régression linéaire détaillées dans le tableau II et les figures 5 et 6 .

Fig. 3. Comparison for the trimester 15 June-15 September of (1) the average value of temperatures continuously measured in the Loire upstream of the Dampierre nuclear power plant (series 2); (2) the average values of the three-hourly temperatures calculated by CALNAT et COURNAT; (3) the average value of the three-hourly air temperatures measured at the Météo-France station of Tours (years 1980-2003). Details on the regression lines are specified in Table II and in figures 5 and 6. 

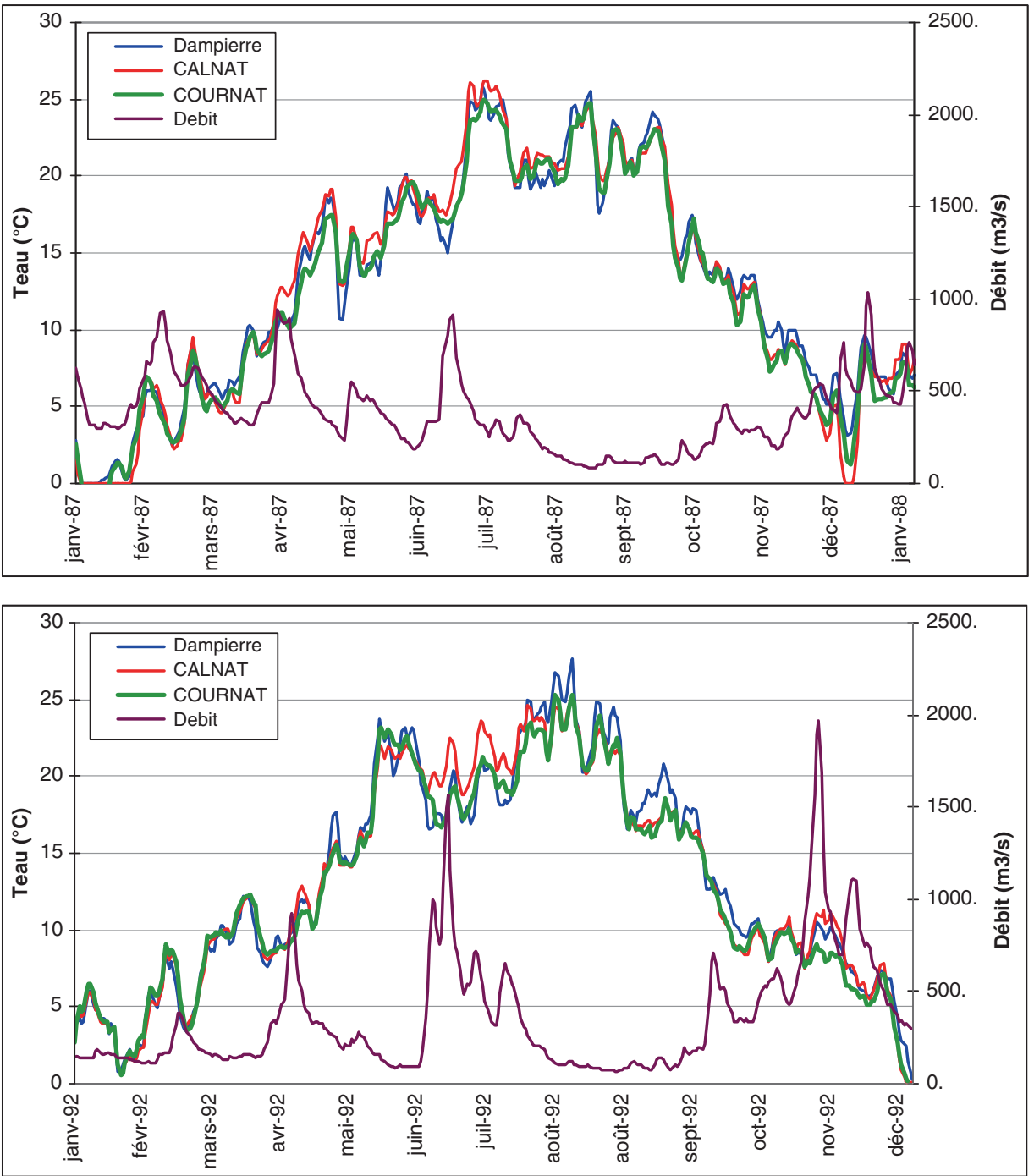

Fig. 4. Comparaison de la performance de CALNAT et COURNAT pour simuler la température de la Loire moyenne pour deux années à crue estivale (1987 et 1992).

Fig. 4. Comparison of CALNAT and COURNAT ability to reproduce the mid Loire temperature for two years with summer flood (1987 and 1992).

pour les 4 paramètres atmosphériques favorisent toutes un échauffement de l'eau. Le tableau II synthétise les résultats obtenus.
II apparaît ainsi que la tendance d'échauffement moyen calculé par CALNAT sur les 24 ans $\left(1,55^{\circ} \mathrm{C}\right)$ représente $84 \%$ de la tendance 
a

\begin{tabular}{|c|c|c|c|}
\hline $\mathrm{T}$ eau trimestrie & e ar & it (mi & \\
\hline Tendance & 0.0777 & ${ }^{\circ} \mathrm{C} /$ an & \\
\hline Significativité & $99.6 \%$ & & \\
\hline & Médian & IC Binf $90 \%$ & IC Bsup $90 \%$ \\
\hline Variation sur la période $1980-2003$ & 1.86 & 0.92 & 2.80 \\
\hline
\end{tabular}

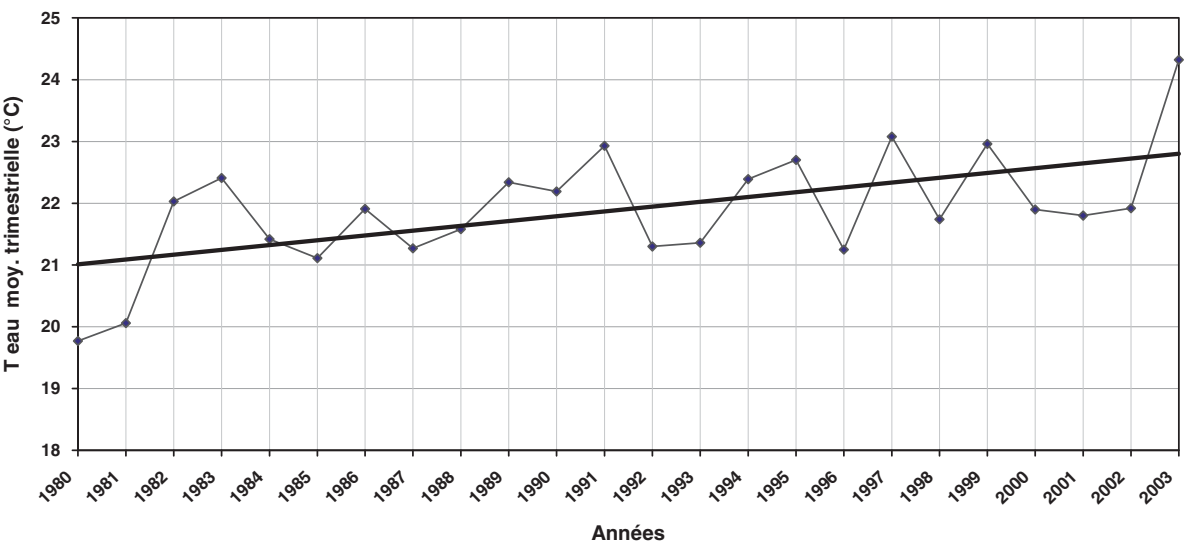

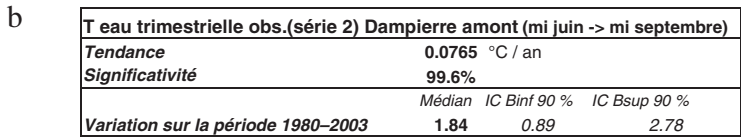

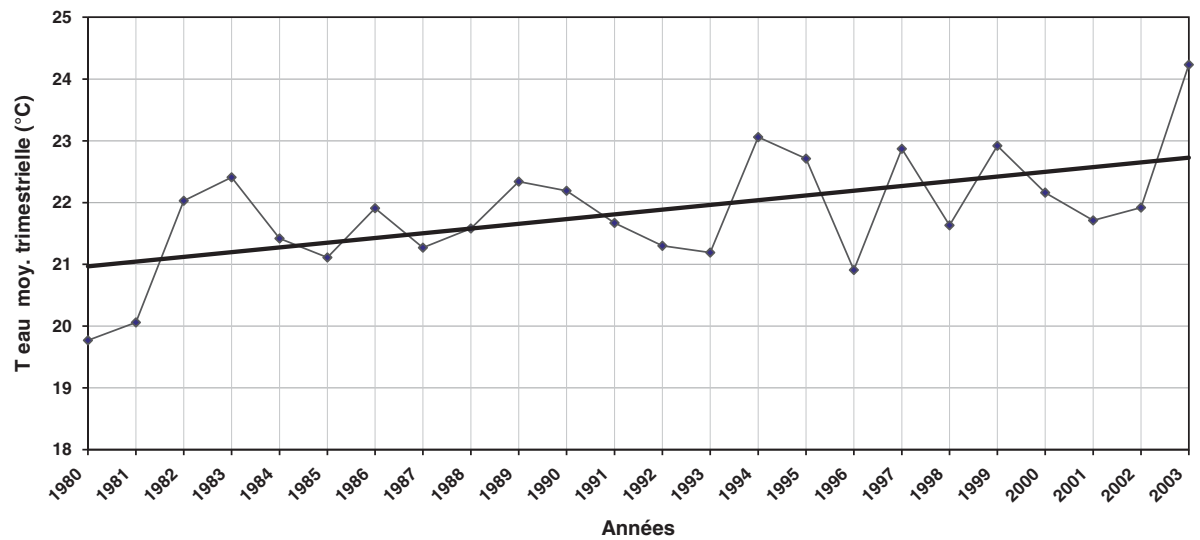

Fig. 5. Résultats du test de régression linéaire appliqué aux moyennes trimestrielles (15 juin15 septembre) des enregistrements en continu de températures de Loire aux sites Dampierre amont et Belleville amont et des résultats de trois modèles thermiques : CALNAT, COURNAT et modèle statistique STAT3 (années 1980 à 2003).

Fig. 5. Results of the linear regression test applied to the quarterly (15 June-15 September) averages of continuous recordings of the Loire temperature at "Dampierre amont" and "Belleville amont" sites and to the quarterly average results of three thermal models: CALNAT, COURNAT and statistical model STAT3 (years 1980-2003). 


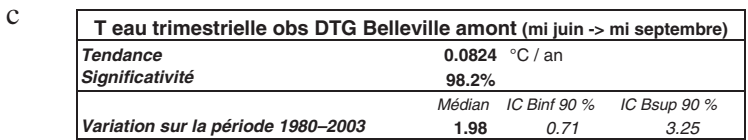

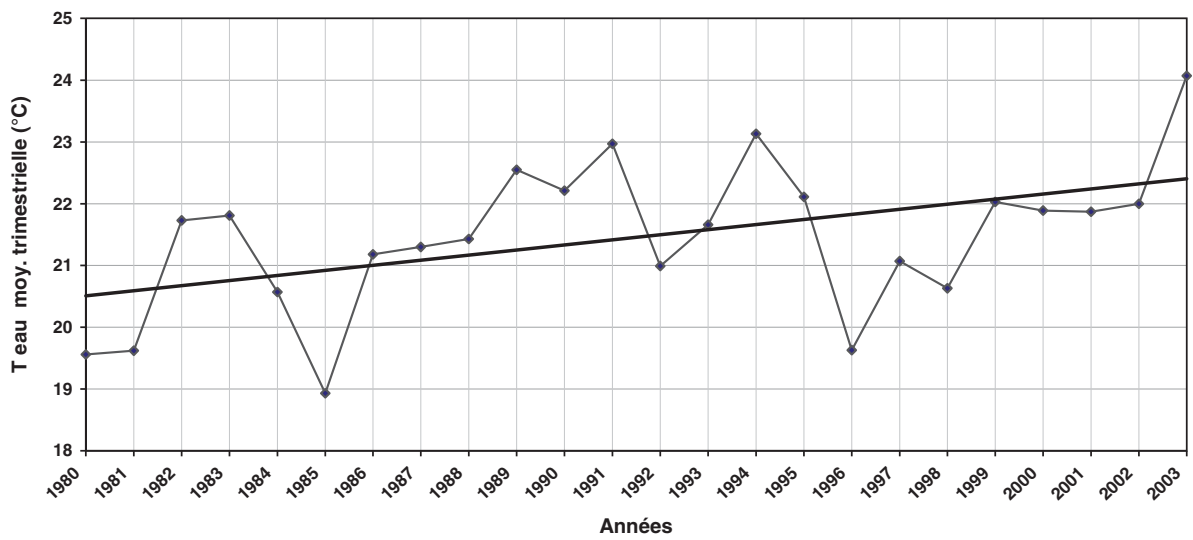

d

\begin{tabular}{|c|c|c|}
\hline \multicolumn{3}{|c|}{ T eau trimestrielle modèle CALNAT (mi juin -> mi septembre) } \\
\hline Tendance & $0.0649{ }^{\circ} \mathrm{C} / \mathrm{an}$ & \\
\hline Significativité & $98.7 \%$ & \\
\hline & Médian IC Binf $90 \%$ & IC Bsup $90 \%$ \\
\hline Variation sur la période $1980-2003$ & 0.61 & 2.50 \\
\hline
\end{tabular}

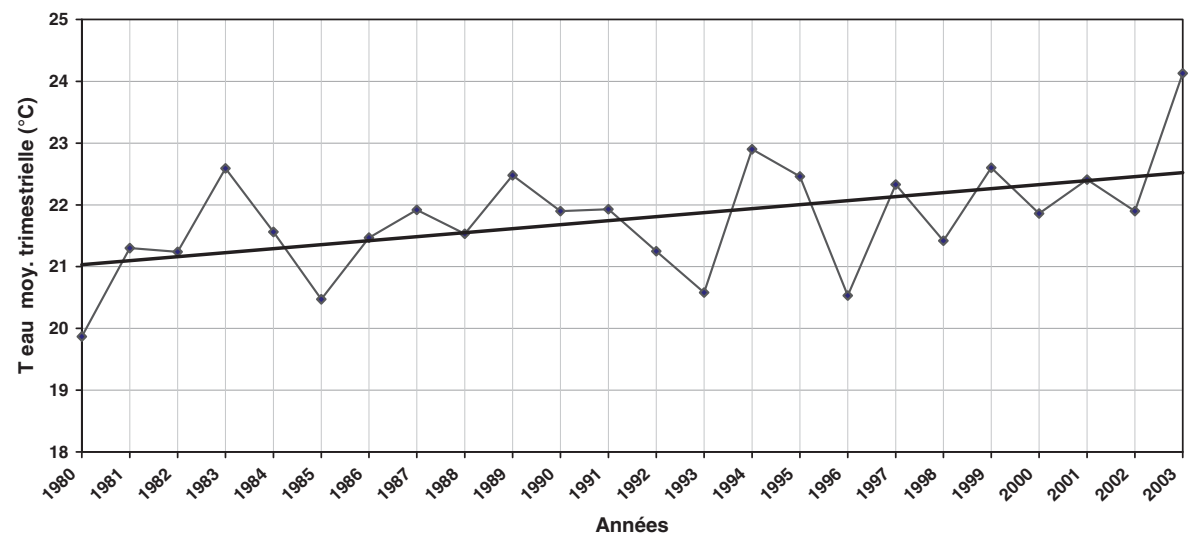

Fig. 5. Suite.

Fig. 5. Continued. 
Analyse de la temperature de la Loire moyenne en été sur la période 1949 à 2003

247

e

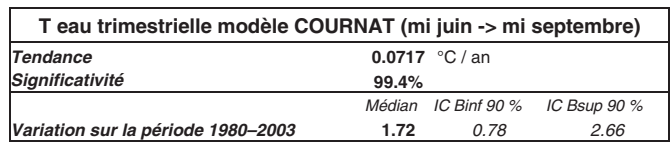

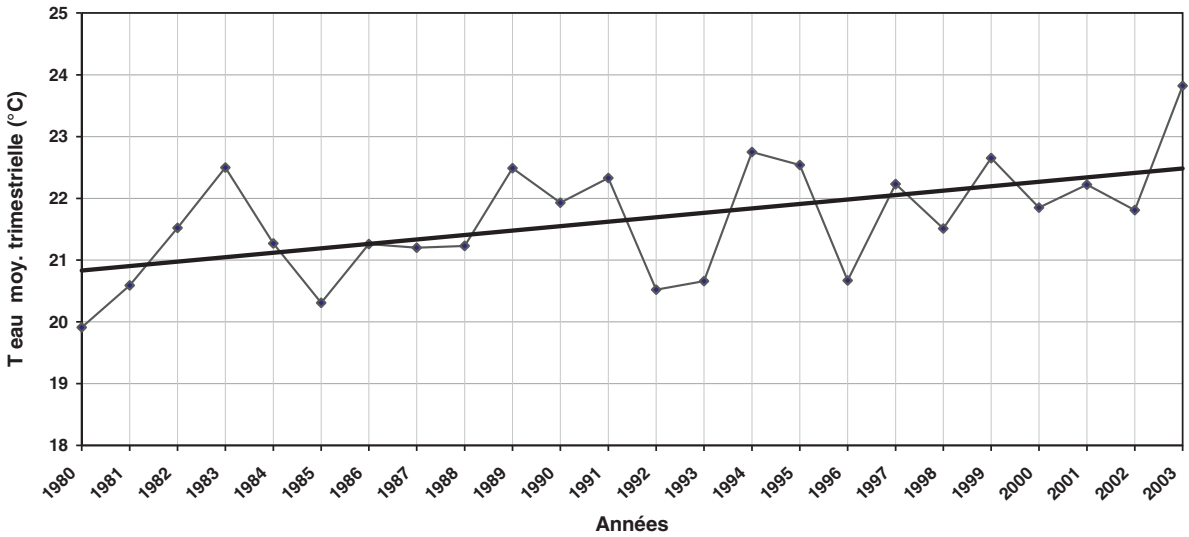

f

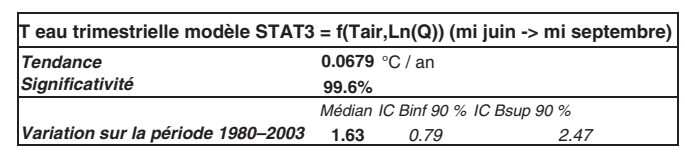

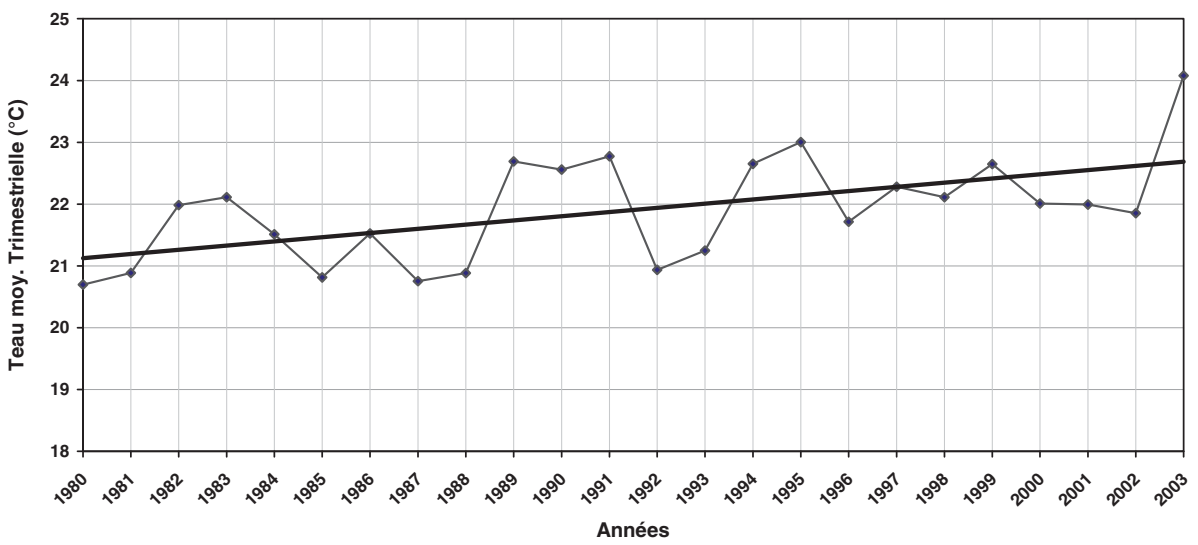

Fig. 5. Suite.

Fig. 5. Continued. 


\begin{tabular}{|l|lcll|}
\hline \multicolumn{4}{|c|}{ T air trimestrielle mesures } & Tours (mi juin -> mi septembre) \\
\hline Tendance & $\mathbf{0 . 0 6 3 0}$ & ${ }^{\circ} \mathrm{C} /$ an \\
Significativité & $\mathbf{9 7 . 8 \%}$ & \\
\hline & Médian & IC Binf $90 \%$ & IC Bsup $90 \%$ \\
Variation sur la période & $\mathbf{1 9 8 0 - 2 0 0 3}$ & $\mathbf{1 . 5 1}$ & 0.50 & 2.52 \\
\hline
\end{tabular}

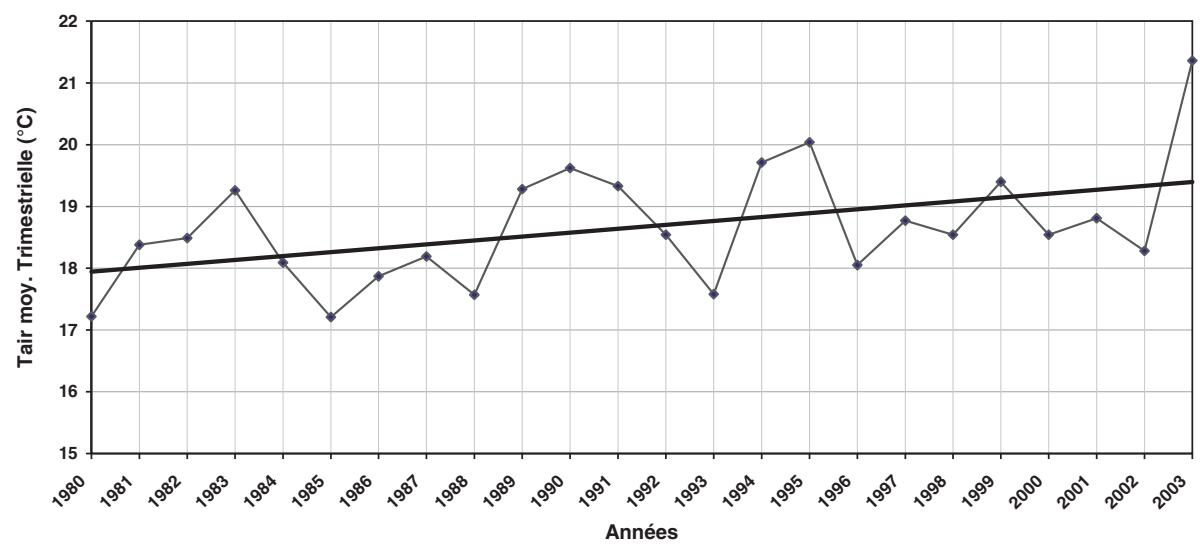

b

\begin{tabular}{|lccc|}
\hline Vitesse vent & trimestrielle mesures Tours (mi juin -> mi septembre) \\
\hline Tendance & -0.0162 & $(\mathrm{~m} / \mathrm{s}) /$ an \\
Significativité & $\mathbf{9 8 . 8 \%}$ & & \\
\hline & Médian & IC Binf $90 \%$ & IC Bsup $90 \%$ \\
Variation sur la période $1980-2003$ & -0.39 & -0.62 & -0.15 \\
\hline
\end{tabular}

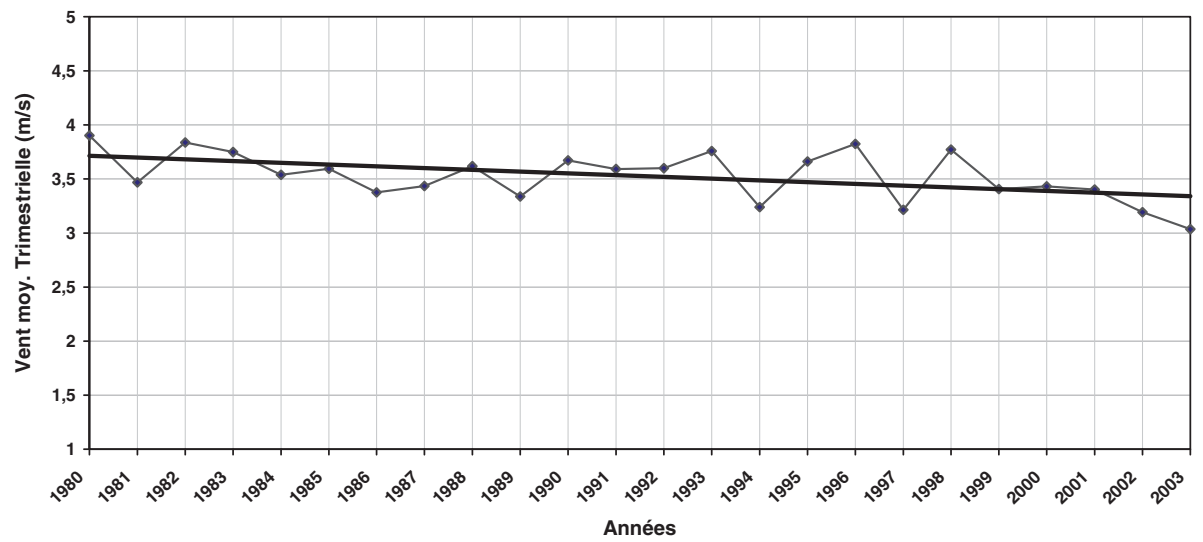

Fig. 6. Résultats du test de régression linéaire appliqué aux moyennes trimestrielles (15 juin15 septembre) de quatre paramètres atmosphériques relevés à la station Météo-France de Tours (température d'air, pression de vapeur d'eau, vitesse de vent à 10 mètres, et nébulosité du ciel) et du débit de la Loire à Gien (années 1980-2003).

Fig. 6. Results of the linear regression test applied to the quarterly (15 June-15 September) averages of (1) four atmospheric parameters measured at Tours meteorological station (air temperature, steam pressure, wind velocity at $10 \mathrm{~m}$ and sky nebulosity); (2) the river discharge at Gien (years 1980-2003). 
Analyse de la température de la Loire moyenne en été sur la période 1949 à 2003

249

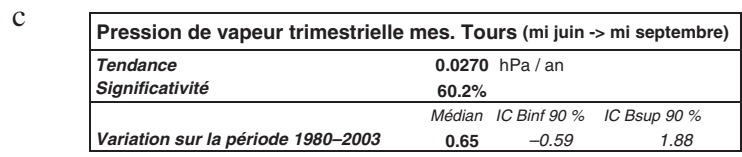

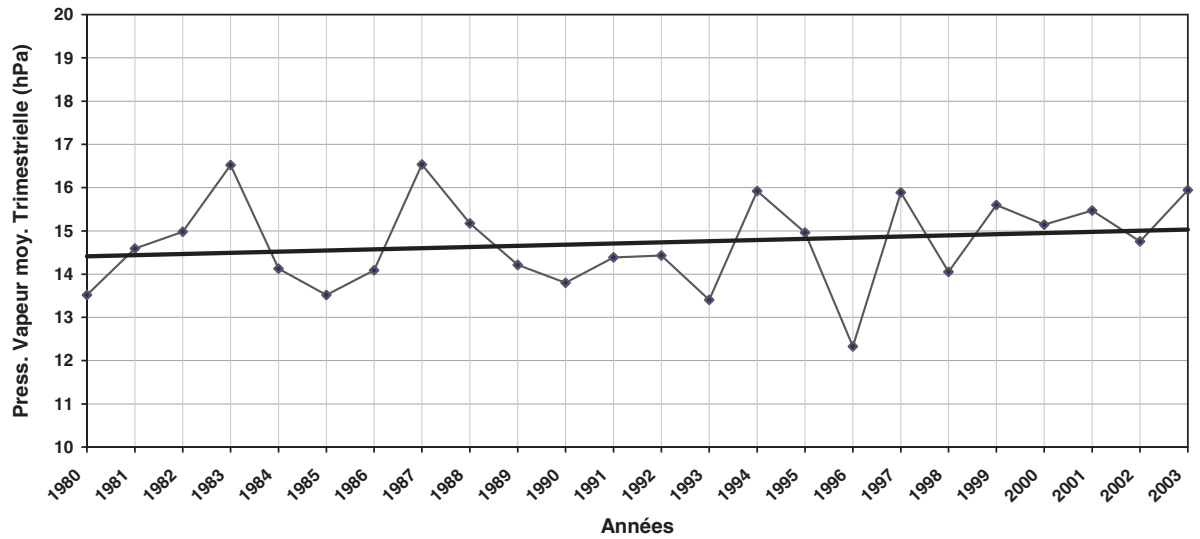

d

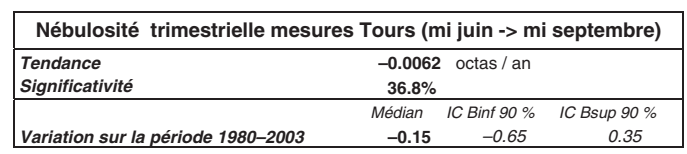

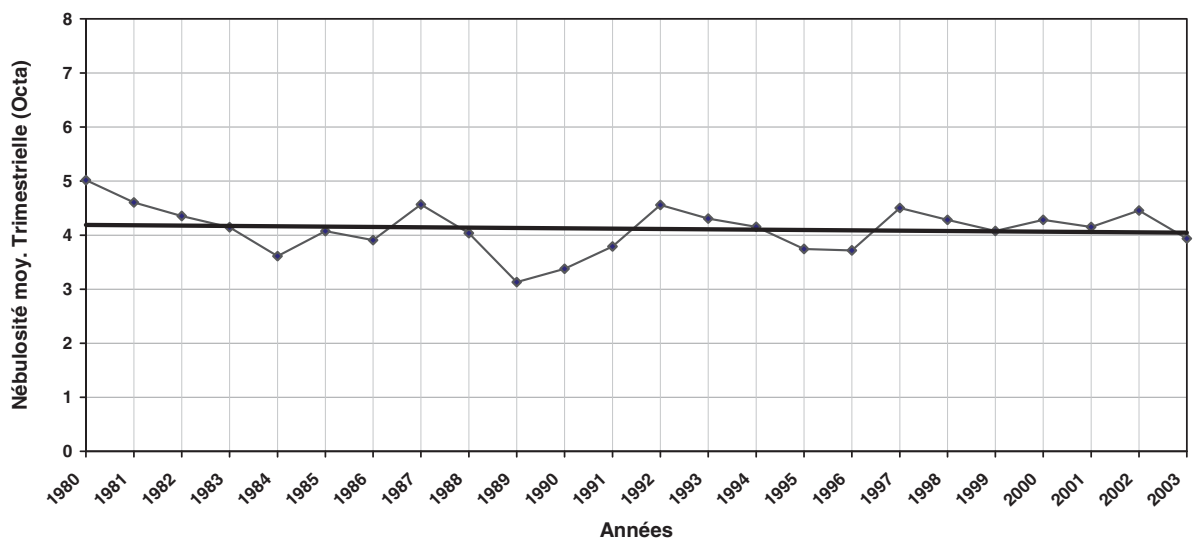

Fig. 6. Suite.

Fig. 6. Continued. 


\begin{tabular}{|l|lccc|}
\hline \multicolumn{4}{|c|}{ Débit trimestriel mesures Gien (mi juin $\rightarrow$ mi septembre) } \\
\cline { 2 - 5 } & Tendance & -0.0248 & $\mathrm{Ln}(\mathrm{m} 3 / \mathrm{s}) /$ an \\
& $97.3 \%$ & & \\
\cline { 2 - 3 } & Médian & IC Binf $90 \%$ & IC Bsup $90 \%$ \\
\hline & -69.6 & -121 & -21 \\
\hline
\end{tabular}

N.B. : la variation est donnée sur la variable débit tandis que la tendance est analysée sur la variable $\ln (Q)$ pour respecter

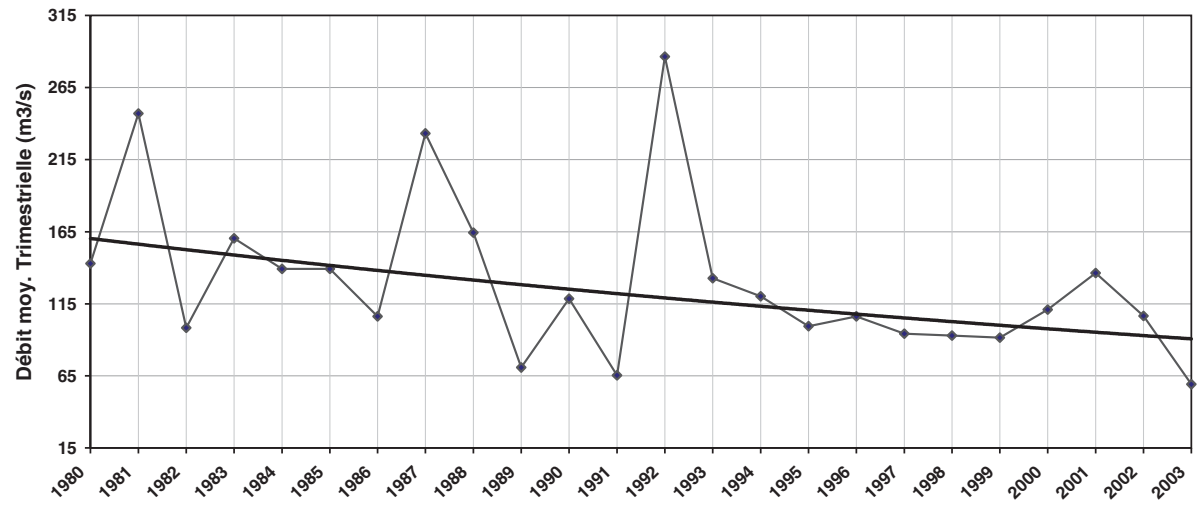

Années

Fig. 6. Suite.

Fig. 6. Continued.

Tableau II. Test de régression linéaire appliqué sur la période 1980-2003 aux moyennes trimestrielles 15 juin-15 septembre de six paramètres mesurés sur la période et de résultats de trois modèles de température d'eau : CALNAT, COURNAT et modèle statistique de régression multilinéaire STAT3 ayant la température trimestrielle d'air à Tours et le logarithme népérien du débit trimestriel à Gien comme variables explicatives.

Table II. Linear regression test applied to quarterly (15 June-15 September) averages of (1) six measured parameters; (2) results of three water temperature models: CALNAT, COURNAT and multilinear regression statistical model STAT3 with the quarterly air temperature at Tours and the Neperian logarithm of the quarterly river discharge at Gien as explaining variables (years 1980-2003).

\begin{tabular}{|c|c|c|c|c|c|}
\hline Météo et Hydrologie & Unité & Variation (unitél(1980-2003)) & IC Binf. $90 \%$ & IC Bsup. $90 \%$ & Significativité \\
\hline Température d'air Tours & ${ }^{\circ} \mathrm{C}$ & 1.51 & 0,50 & 2.52 & $97.8 \%$ \\
\hline Pression de vapeur d'eau Tours & $\mathrm{nPa}$ & 0.65 & -0.59 & 1.88 & $60.2 \%$ \\
\hline Vitesse de vent $10 \mathrm{~m}$ Tours & $\mathrm{m} / \mathrm{s}$ & -0.38 & -0.62 & -0.15 & $98.8 \%$ \\
\hline Nebulosité Tours & octa & -0.15 & -0.65 & 0.35 & $36.8 \%$ \\
\hline Débit Gien & $\mathrm{m} 3 / \mathrm{s}$ & -69.6 & -121 & -21 & $97.3 \%$ \\
\hline Température d'eau & Unité & Variation $\left({ }^{\circ} \mathrm{C} /(1980-2003)\right)$ & IC Binf. $90 \%$ & IC Bsup. $90 \%$ & Significativité \\
\hline Mesures Dampierre amont (série 1) & ${ }^{\circ} \mathrm{C}$ & 1.86 & 0,92 & 2.80 & $99.6 \%$ \\
\hline modèle stat. STAT3 $=f(T a i r, \ln (Q))$ & ${ }^{\circ} \mathrm{C}$ & 1.63 & 0.79 & 2,47 & $99,6 \%$ \\
\hline CALNAT & ${ }^{\circ} \mathrm{C}$ & 1.56 & 9.61 & 2.50 & $98.7 \%$ \\
\hline COURNAT & ${ }^{\circ} \mathrm{C}$ & 1.72 & 0.78 & 2.66 & $99.4 \%$ \\
\hline
\end{tabular}


d'échauffement dégagée des mesures à la station Dampierre amont ${ }^{(5)}$ $\left(1,85^{\circ} \mathrm{C}\right)$, une tendance comparable $\left(2,0^{\circ} \mathrm{C}\right)$ étant obtenue à la station Belleville amont située près de trente kilomètres en amont. Cela montre à la fois les capacités du code CALNAT à reproduire assez correctement des tendances observées en Loire moyenne à l'échelle de décennies, et l'effet prépondérant des variations des conditions atmosphériques dans l'explication de l'échauffement de la Loire moyenne sur ces 24 ans, puisque ce sont les seules entrées de CALNAT qui varient au cours du temps.

Pour estimer la part respective des 4 paramètres atmosphériques dans la tendance d'échauffement calculée, on réalise quatre séries de deux simulations complémentaires de CALNAT permettant de révéler la sensibilité du modèle à ces données d'entrée. À partir des variations constatées sur la période 1980-2003 à l'échelle trimestrielle $\left(\Delta \mathrm{T}_{\text {air 1980-2003 }}, \Delta \mathrm{T}_{\text {vap1980-2003 }}\right.$, $\ldots)$, on réalise pour chaque paramètre atmosphérique deux séries de données météorologiques fictives. Ces séries conservent les valeurs observées pour les autres paramètres atmosphériques et définissent à chaque pas tri-horaire le paramètre modifié de la façon suivante, illustrée ici pour la température d'air :

- $\mathrm{T}_{\text {air1 }}=\mathrm{T}_{\text {airObs }}+\Delta \mathrm{T}_{\text {air1980-2003 }}$ pour la première série;

- $\mathrm{T}_{\text {air2 }}=\mathrm{T}_{\text {airObs }}-\Delta \mathrm{T}_{\text {air1980-2003 }}$ pour la deuxième série.

L'exploitation par CALNAT de ces deux jeux de données conduit à deux calculs par paramètre atmosphérique

(5) Des tendances très proches d'échauffement de la Loire sur les 24 ans sont obtenues avec les séries 1 et 2 de Dampierre amont (médianes de $1,86^{\circ} \mathrm{C}$ et $1,84^{\circ} \mathrm{C}$ respectivement). dont on extrait les moyennes $M_{1}$ et $M_{2}$ des températures trimestrielles d'eau sur les 24 ans. Ces valeurs $M_{1}$ et $M_{2}$ sont comparées à la moyenne $\mathrm{M}_{\text {ref }}$ obtenue sur la même période à partir de la simulation de CALNAT utilisant les observations atmosphériques réelles. L'échauffement de l'eau $\Delta \mathrm{T}_{\text {eau }}$ évalué avec CALNAT pour chaque modification de paramètre atmosphérique est évalué par l'équation : $\Delta T_{\text {eau }}=\left(\left(M_{1}-\right.\right.$ $\left.\left.M_{\text {ref }}\right)+\left(M_{\text {ref }}-M_{2}\right)\right) / 2=\left(M_{1}-M_{2}\right) / 2$.

La première série de simulations ajoute ou retire $1,5^{\circ} \mathrm{C}$ aux températures d'air mesurées à Tours; la deuxième ajoute ou retire $0,65 \mathrm{hPa}$ aux pressions de vapeur d'eau ${ }^{(6)}$; la

(6) La masse de d'eau rapportée à l'unité de masse d'air sec ( $\mathrm{kg}$ d'eau / kg d'air air sec) peut être approchée par l'équation $0,622 \mathrm{P}_{\mathrm{vap}} / \mathrm{P}_{\mathrm{atm}}$ qui indique que la pression partielle de la vapeur d'eau $P_{\text {vap }}$ qui est une entrée de CALNAT et COURNAT est un témoin de quantité de vapeur d'eau dans l'air. La tendance statistique à l'augmentation de $0,65 \mathrm{hPa}$ du niveau moyen de $P_{\text {vap }}$ en été (15 juin-15 septembre) sur la période 1980-2003 caractérise donc un accroissement de la quantité de vapeur d'eau dans la masse d'air. II peut paraître paradoxal que dans le même temps l'humidité relative HR - paramètre de caractérisation de la vapeur d'eau plus connu du grand public - qui est le rapport de la masse de vapeur d'eau à la masse d'eau qui serait contenue à saturation et s'écrit $\mathrm{HR}=$ $100 \mathrm{P}_{\text {vap }} / \mathrm{P}_{\text {vap }}^{\text {sat }}$ présente simultanément une tendance de diminution de 2,7\%. Cela est dû au fait que l'élévation statistique simultanée de la température d'air d'un niveau moyen de $18,5^{\circ} \mathrm{C}$ vers un niveau moyen de $20^{\circ} \mathrm{C}$ conduit à une augmentation proche de $2 \mathrm{hPa}$ du niveau moyen de la pression de vapeur d'eau à saturation $\mathrm{P}_{\text {vap }}^{\text {sat }}$. Cette augmentation de $2 \mathrm{hPa}$ est suffisamment élevée pour conduire à une diminution du rapport $\mathrm{P}_{\text {vap }} / \mathrm{P}_{\text {vap }}^{\text {sat }}$, sachant que le niveau moyen de $P_{\text {vap }}$ est proche de $15 \mathrm{hPa}$. On note en passant que $P_{\text {vap }}$ est un indicateur quantitatif de vapeur d'eau plus indépendant de la température d'air que HR, qui l'inclut explicitement via la formulation de $\mathrm{P}_{\text {vap }}^{\text {sat }}$. 


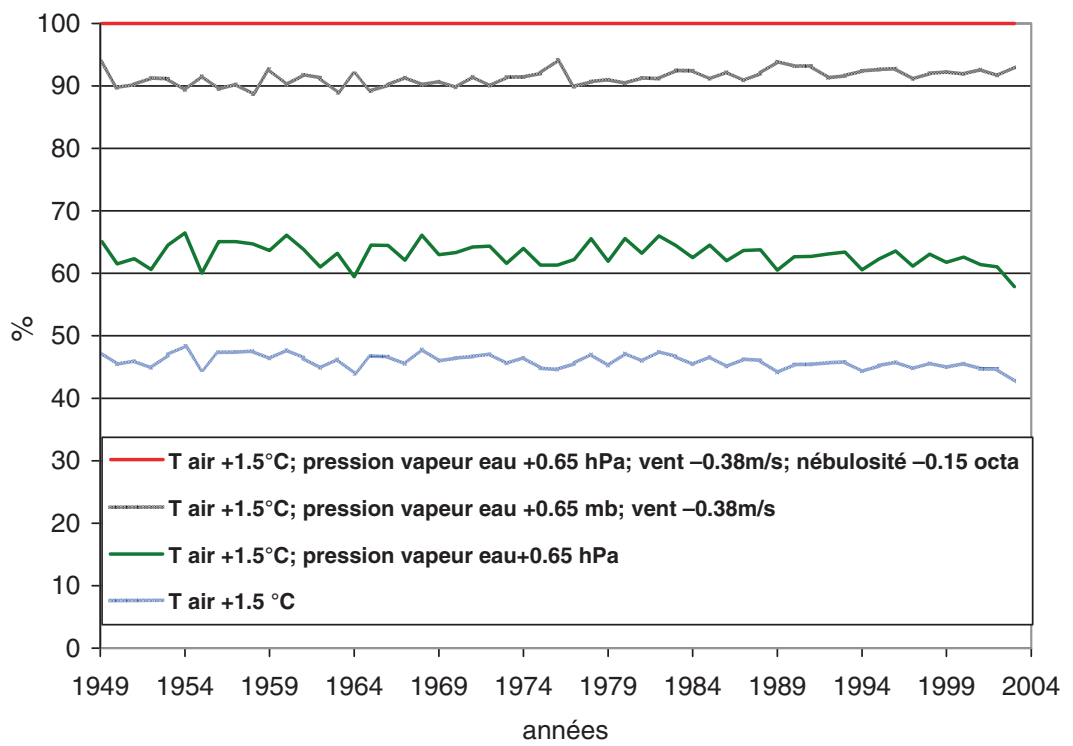

Fig. 7. Estimation de la part respective des tendances d'évolution de la température d'air, de la pression de vapeur d'eau, de la vitesse de vent et de la nébulosité du ciel sur la période 1980-2003 (trimestre 15 juin-15 septembre) dans les échauffements d'eau calculés par CALNAT.

Fig. 7. Estimate of the respective contribution of air temperature, steam pressure, wind velocity and sky nebulosity trends over 1980-2003 (trimester 15 June-15 September) to water warming calculated by CALNAT.

troisième retire ou ajoute $0,38 \mathrm{~m} / \mathrm{s}$ en moyenne ${ }^{(7)}$ à la vitesse de vent, et la quatrième retire ou ajoute 0,15 octa en moyenne à la nébulosité. Les résultats trouvés pour $\Delta T_{\text {eau }}$ sont résumés dans le tableau III. Sachant que les

(7) Pour retirer $0,38 \mathrm{~m} / \mathrm{s}$ de vent en moyenne dans la simulation de la troisième série, la vitesse de vent observée est diminuée de $0,39 \mathrm{~m} / \mathrm{s}$ si elle est supérieure ou égale à ce seuil et mise à zéro dans le cas inverse. Pour retirer 0,15 octa en moyenne dans la quatrième série de simulations, la nébulosité observée est diminuée de 0,18 octa si elle est supérieure ou égale à 1, et n'est pas modifiée si elle est déjà égale à zéro. De la même façon pour ajouter 0,15 octa en moyenne, la nébulosité observée est augmentée de 0,165 octa si elle est inférieure ou égale à 7 , et n'est pas modifiée si elle est égale à 8 . tendances réelles d'évolution des 4 paramètres atmosphériques sur les 24 ans vont toutes dans le sens d'un échauffement de l'eau, cette approche permet d'estimer (Fig. 7) que l'échauffement tendanciel trimestriel calculé sur les 24 ans par CALNAT est dû pour près de $46 \%$ à l'augmentation de la température d'air ${ }^{(8)}, 28 \%$ à la baisse de la vitesse de vent, $18 \%$ à

(8) Gosse et al. (1996) pour la température d'air, la vitesse de vent et la nébulosité du ciel et Dupeyrat et Berthier (2006) pour la température d'air ont déjà montré l'effet d'un ajout/retrait artificiel de valeur à un paramètre météorologique sur les résultats de CALNAT au cours de l'année. Les ordres de grandeur trouvés sont similaires. 
l'augmentation de la pression de vapeur d'eau et $8 \%$ à la baisse de la nébulosité du ciel. Avec cette méthode qui vise à dissocier l'effet des paramètres atmosphériques et répartit uniformément un delta constant sur des paramètres atmosphériques à chaque pas tri-horaire, seulement $14 \%$ de l'échauffement tendanciel calculé sur 24 ans (soit $0,22^{\circ} \mathrm{C}$ sur les $1,55^{\circ} \mathrm{C}$ ) n'est pas reproduit.

Le tableau III confirme les constatations antérieures (Gosse et al., 1996) d'une bonne symétrie des résultats de CALNAT avec ajout ou retrait d'une variation modérée de paramètre atmosphérique. On aurait ainsi trouvé des résultats très proches - à $1 \%$ près sur la part relative de chaque paramètre atmosphérique en ne faisant qu'un seul type de simulation consistant à ajouter (ou à retirer) à chaque paramètre atmosphérique la tendance observée pour ce dernier. La figure 7 montre par ailleurs que la considération d'un plus grand jeu de données météorologiques (1949-2003) ne change pas les parts relatives des paramètres atmosphériques dans l'explication des évolutions de la température de l'eau.

On constate (Tab. II et Fig. 5) qu'il est possible avec COURNAT de retrouver $92 \%$ (soit $1,7^{\circ} \mathrm{C}$ pour $1,85^{\circ} \mathrm{C}$ ) de la tendance d'échauffement observée à Dampierre sur 1980-2003.

COURNAT permet d'intégrer deux effets de la tendance de baisse de $70 \mathrm{~m}^{3} / \mathrm{s}$ du débit moyen à Gien sur ces 24 ans pour le trimestre 15 juin15 septembre : moindre profondeur de l'écoulement et diminution de la vitesse d'écoulement qui atténue le signal thermique de la condition limite amont, avec une influence dans les situations à haut débit.

Le premier effet est vraisemblablement modéré : on remarque en effet (Fig. 8) que le remplacement dans CALNAT de la profondeur d'inertie thermique constante à 2 mètres par celle de COURNAT $\left(\mathrm{H}=0,161 \mathrm{Q}^{0,453}\right)$ ou par la profondeur moyenne de la Loire sur le tronçon Gien-Dampierre

Tableau III. Effet sur l'évolution de la moyenne des températures trimestrielles (15 juin-15 septembre) de l'eau sur la période 1980-2003 d'une modification des valeurs tri-horaires de paramètres atmosphériques observés à Tours. La modification appliquée est l'ajout ou le retrait de la variation tendancielle sur 1980-2003 de la moyenne trimestrielle d'un paramètre atmosphérique. Le modèle CALNAT est utilisé pour les calculs.

Table III. Impact on the average of the quarterly (15 June-15 September) water temperatures over 1980-2003 of a modification, every three hours, of the atmospheric parameters observed at Tours. The modification is the addition or substraction of the variation over 1980-2003 of the quarterly averages of an atmospheric parameter. The model CALNAT is used.

\begin{tabular}{|c|c|c|c|c|}
\hline \multirow[b]{2}{*}{ Variable } & \multirow{2}{*}{$\begin{array}{c}\text { Variation évaluée } \\
\text { (15 juin - } 15 \text { septembre) } \\
\text { sur la période } \\
1980-2003\end{array}$} & \multicolumn{3}{|c|}{$\begin{array}{c}\text { effet sur moyenne trimestrielle CALNAT } \\
15 \text { juin- } 15 \text { septembre } \\
\text { (moyenne sur } 1980-2003 \text { en }{ }^{\circ} \mathrm{C} \text { ) }\end{array}$} \\
\hline & & M1 - Mref & Mref - M2 & $\Delta$ Teau \\
\hline Température d 'air & $1.5^{\circ} \mathrm{C}$ & 0,615 & 0,62 & 0,62 \\
\hline Pression de vapeur d'eau & $0,65 \mathrm{hPa}$ & 0,24 & 0,24 & 0,24 \\
\hline Vitesse de vent & $-0,38 \mathrm{~m} / \mathrm{s}$ & 0,39 & 0,35 & 0,37 \\
\hline nébulosité du ciel & -0.15 octa & 0,11 & 0,095 & 0,10 \\
\hline
\end{tabular}




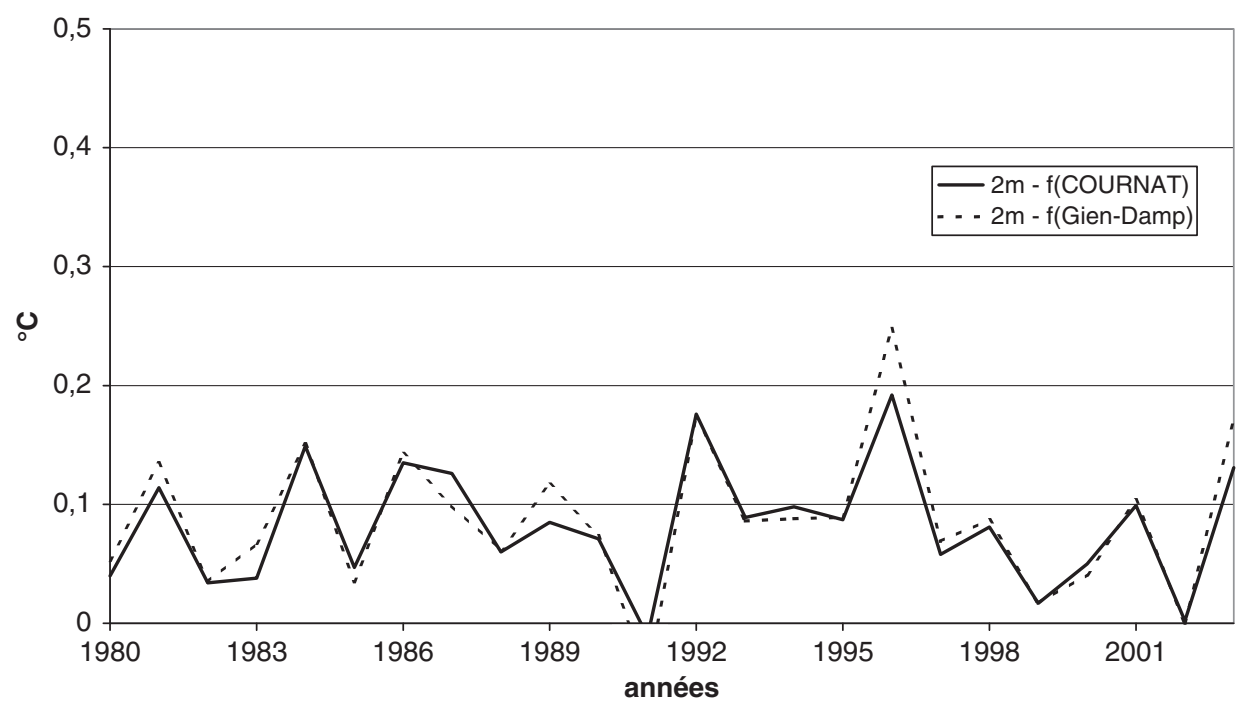

Fig. 8. Effet d'un changement de la profondeur d'inertie thermique sur les moyennes trimestrielles (15 juin-15 septembre) de température d'eau calculées par CALNAT.

Fig. 8. Impact of a change of the inertial thermal depth on the quarterly (15 June-15 September) averages of water temperature calculated by CALNAT.

décrite par l'équation ${ }^{(9)} \mathrm{H}=0,102 \mathrm{Q}^{0,49}$ modifie très peu (moins de $0,1^{\circ} \mathrm{C}$ en moyenne) les moyennes trimestrielles de température d'eau calculées, les tendances d'échauffement sur 24 ans pour le trimestre 15 juin- 15 septembre s'écartant de moins de $0,01^{\circ} \mathrm{C}$.

Le deuxième effet est a priori plus important. On constate en particulier (Fig. 9) une tendance à la diminution de la fréquence de dépassement du seuil de débit de $300 \mathrm{~m}^{3} / \mathrm{s}$ au cours des 24 ans sur ce trimestre 15 juin-15 septembre. Cette baisse

(9) C'est l'équation réactualisée en 2000 par EDF R\&D pour le tronçon Gien-Dampierre dans le cadre de la mise au point d'un modèle radioécologique de la Loire, avec $\mathrm{Q}$ débit à Gien en $\mathrm{m}^{3} / \mathrm{s}$ (Siclet et al., 2001). tendancielle des hauts débits en été va par ailleurs dans le sens de l'allongement de la période pendant laquelle la Loire moyenne est à la température naturelle locale correspondant aux conditions de validité de CALNAT.

La différence entre les tendances d'échauffement de COURNAT qui considère le débit et de CALNAT qui n'en tient pas compte, est de $0,16^{\circ} \mathrm{C}$, soit $8,5 \%$ de la tendance d'échauffement de la Loire à Dampierre. Cet écart ne correspond pas cependant à l'influence stricte du débit car COURNAT ne répond pas de la même manière que CALNAT à une variation de vitesse de vent à Tours : un calcul montre que le remplacement dans CALNAT des valeurs des paramètres de vent $a$ et $b$ par celles retenues dans COURNAT fait perdre $0,25^{\circ} \mathrm{C}$ 
a

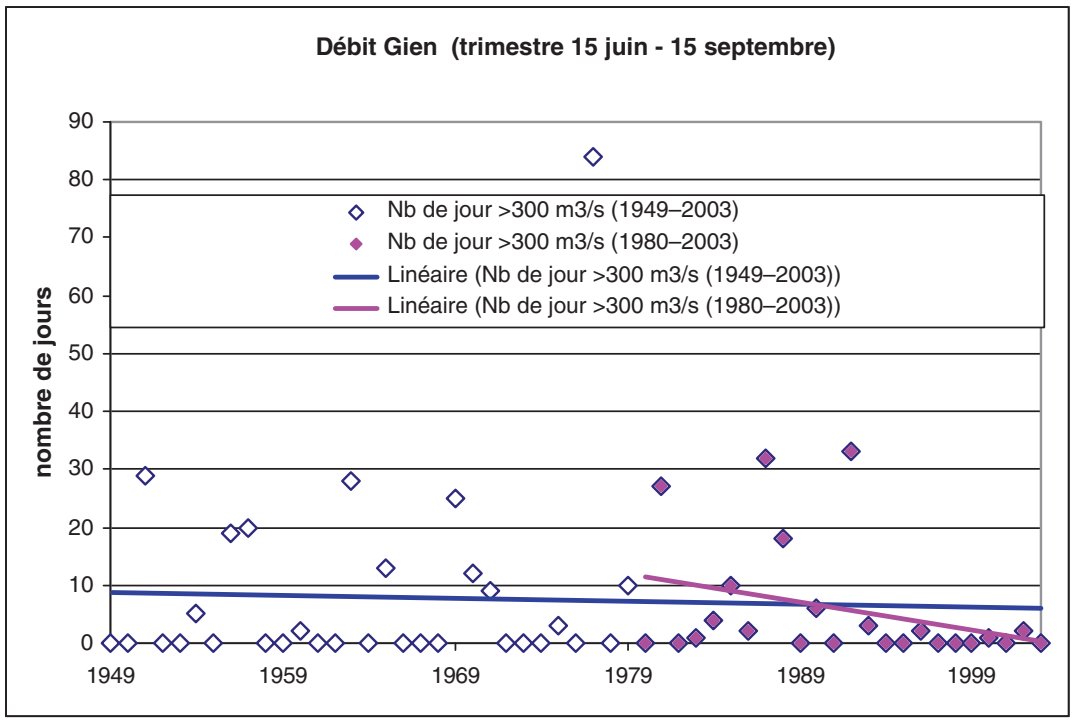

b

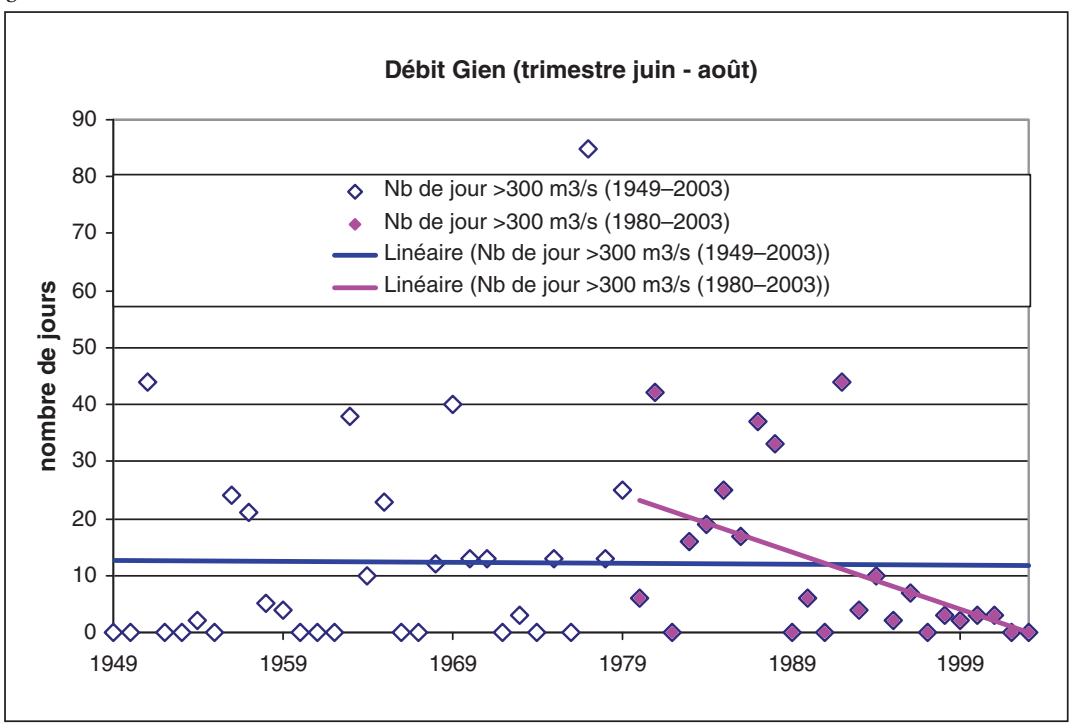

Fig. 9. Nombre de jours des périodes $1^{\text {er }}$ juin-31 août et 15 juin- 15 septembre durant lesquels le débit à Gien a été supérieur à $300 \mathrm{~m}^{3} / \mathrm{s}$.

Fig. 9. Number of days with river discharges greater than $300 \mathrm{~m}^{3} / \mathrm{s}$ at Gien for two quarterly periods (1st June-31 August and 15 June-15 September). 


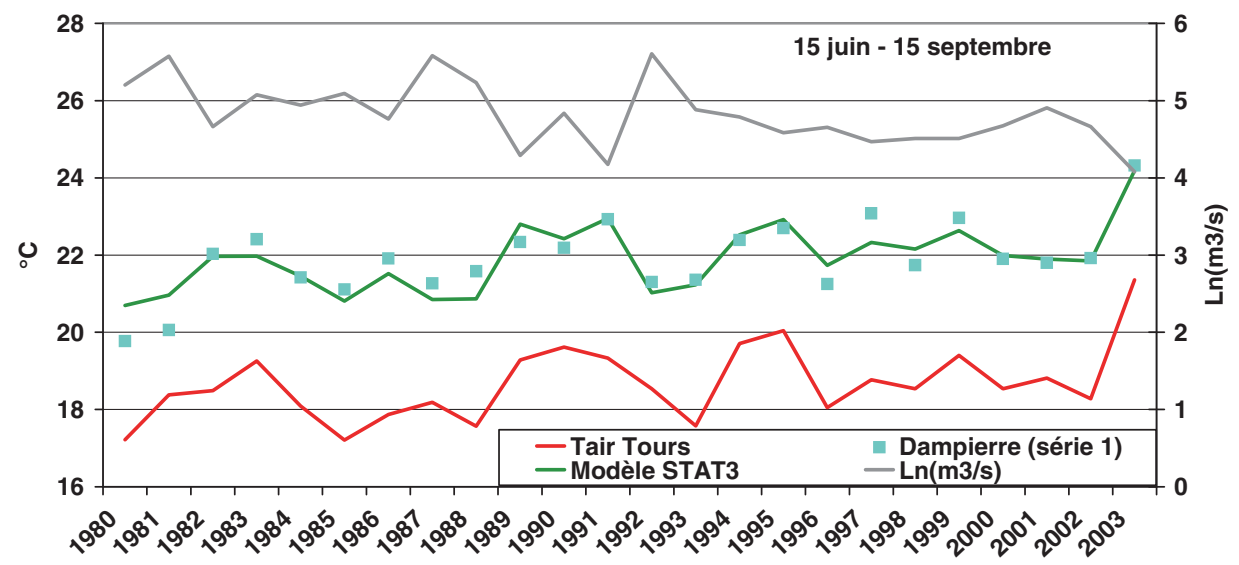

Fig. 10. Comparaison sur le trimestre 15 juin-15 septembre des moyennes des températures de Loire mesurées en continu à la station Dampierre amont (série 1), des températures d'air mesurées à la station Météo-France de Tours, du débit mesuré à Gien et des températures d'eau calculées par un modèle statistique de régression multilinéaire (STAT3) ayant la température trimestrielle d'air à Tours et le logarithme népérien du débit trimestriel à Gien comme variables explicatives (années 1980-2003).

Fig. 10. Comparison for the trimester 15 June-15 September of the average values of continuous measurements of mid-Loire temperature at the Dampierre amont station (series 1), air temperature measurements at the Météo-France station of Tours, Loire discharge measurements at Gien and water temperature calculated by a multilinear regression statistical model (STAT3) taking the quarterly air temperature at Tours and the Neperian logarithm of the quarterly Loire discharge at Gien as explaining variables (years 1980-2003).

à la tendance d'augmentation de la température trimestrielle d'eau calculée par CALNAT ${ }^{(10)}$ sur les 24 ans.

II est donc théoriquement possible que l'évolution du débit ait une influence supérieure à $8 \%$ sur la tendance d'échauffement de l'eau. Une influence maximale de l'ordre de $15 \%$ peut être proposée pour le débit en lui imputant la part d'échauffement de

(10) par ailleurs, le remplacement dans CALNAT de la valeur de e ear par celle retenue par COURNAT a un effet inférieur à $0,01^{\circ} \mathrm{C}$ sur la tendance d'augmentation de la température trimestrielle de l'eau sur les 24 ans. la Loire non représentée par CALNAT sur les 24 ans.

Le modèle statistique STAT3 donne sur les 24 ans (Tab. II, Figs. 3 et 10) une tendance d'échauffement de l'eau de $1,63^{\circ} \mathrm{C}$. II a ainsi une capacité globale de représentation de $88 \%$ de la tendance d'échauffement de la Loire en moyenne trimestrielle sur les 24 ans. À l'aide de l'équation du modèle STAT3, on peut décomposer cette tendance sur la température de l'eau en $0,84^{\circ} \mathrm{C}$ attribuable à l'évolution de la température d'air et $0,79^{\circ} \mathrm{C}$ attribuable à l'évolution du débit : ainsi, dans l'évolution calculée de la température d'eau, $52 \%$ (soit 0,84/1,63) est expliqué par 
la température d'air et $48 \%$ (soit $0,79 / 1,63)$ par le débit.

II apparaît ainsi que le poids donné au débit comparativement aux données atmosphériques locales dans l'explication de l'échauffement de la Loire sur 1980-2003 est nettement plus élevé avec le modèle STAT3. Avec une modélisation statistique utilisant comme variables explicatives deux paramètres non indépendants (ce point est illustré sur la figure 11 qui montre que les étés chauds correspondent généralement à des débits faibles), il est toujours difficile d'interpréter l'influence réelle de chacune des deux variables. Aussi, même si la qualité du modèle statistique peut être jugée tout à fait acceptable pour reproduire les moyennes trimestrielles de température de la Loire moyenne en été sur la chronique de mesures 1976-2003, il semble préférable de considérer des outils déterministes à base physique comme CALNAT et
COURNAT pour analyser des tendances d'évolution à long terme sur cette période de l'année.

\subsection{2 $1^{\text {er juin-31 août }}$}

Cette période a été retenue car elle correspond au trimestre estival que Moatar et Gailhard (2006) ont étudié.

À l'examen de la figure 2 qui montre que l'étiage de la Loire moyenne est centré sur la période 15 juin-15 septembre et de la figure 9 qui compare, sur les trimestres juin-août et 15 juin-15 septembre, l'évolution de la fréquence de dépassement du seuil $300 \mathrm{~m}^{3} / \mathrm{s}$ à Gien, on pressent que le débit va jouer un plus grand rôle d'explication des températures de la Loire moyenne sur le trimestre juinaoût de 1980 à 2003, sous l'effet d'une part d'une plus grande fréquence d'apparition de hauts débits pendant la première quinzaine de juin qui vont

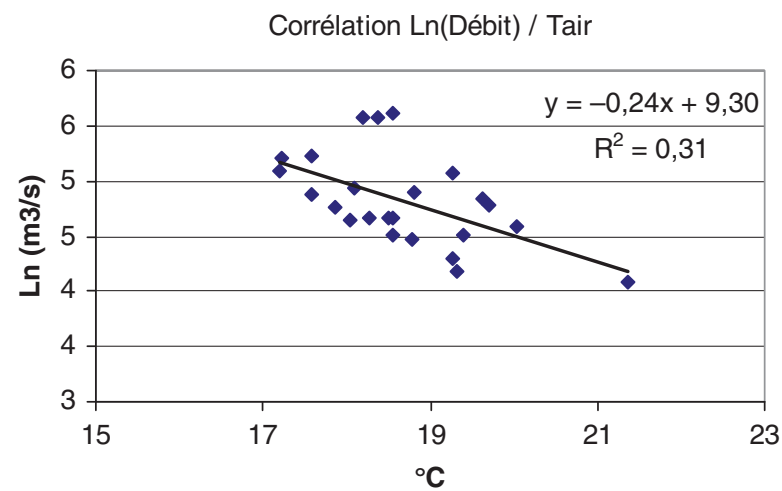

Fig. 11. Relation sur la période 1980-2003 entre la moyenne trimestrielle (15 juin-15 septembre) des températures d'air à Tours et le logarithme népérien de la moyenne trimestrielle des débits à Gien (avec mention de l'équation de régression linéaire Excel).

Fig. 11. Relation between the quarterly ( 15 June- 15 September) average of air temperatures at Tours and the Neperian logarithm of quarterly average of river discharges at Gien over 1980-2003 (the Excel linear regression equation is mentioned). 
renforcer la sensibilité de cette partie du fleuve à des masses d'eau amont peu influencées par le climat local et d'autre part par une diminution marquée de cette fréquence au cours des 24 ans.

L'application de CALNAT, COURNAT et STAT2 sur ce trimestre $1^{\mathrm{er}}$ juin-31 août (Figs. 12 et 13) montre les tendances suivantes sur les 24 ans :

- échauffement moyen calculé par CALNAT : $1,7^{\circ} \mathrm{C}$ soit $74 \%$ de l'échauffement donné par les mesures à Dampierre amont $\left(2,3^{\circ} \mathrm{C}\right)$;

- échauffement moyen calculé par COURNAT : $2,15^{\circ} \mathrm{C}$ soit $93 \%$ de l'échauffement donné par les mesures à Dampierre amont ;
- échauffement moyen calculé par STAT2 : $2,07^{\circ} \mathrm{C}$ soit $90 \%$ de l'échauffement donné par les mesures à Dampierre amont.

Compte tenu du degré de performance de CALNAT, on peut estimer sur le même principe que celui adopté pour la période 15 juin-15 septembre qu'au plus $26 \%$ (part de la tendance d'échauffement non expliquée par CALNAT) de la tendance d'échauffement de la Loire sur 1980-2003 peut être attribué aux variations du débit pour le trimestre juin-août.

Le modèle statistique STAT2 (Fig. 13) donne une tendance d'échauffement de l'eau sur les 24 ans attribuable pour $1,05^{\circ} \mathrm{C}$ à l'évolution de la température d'air et pour $1,02^{\circ} \mathrm{C}$

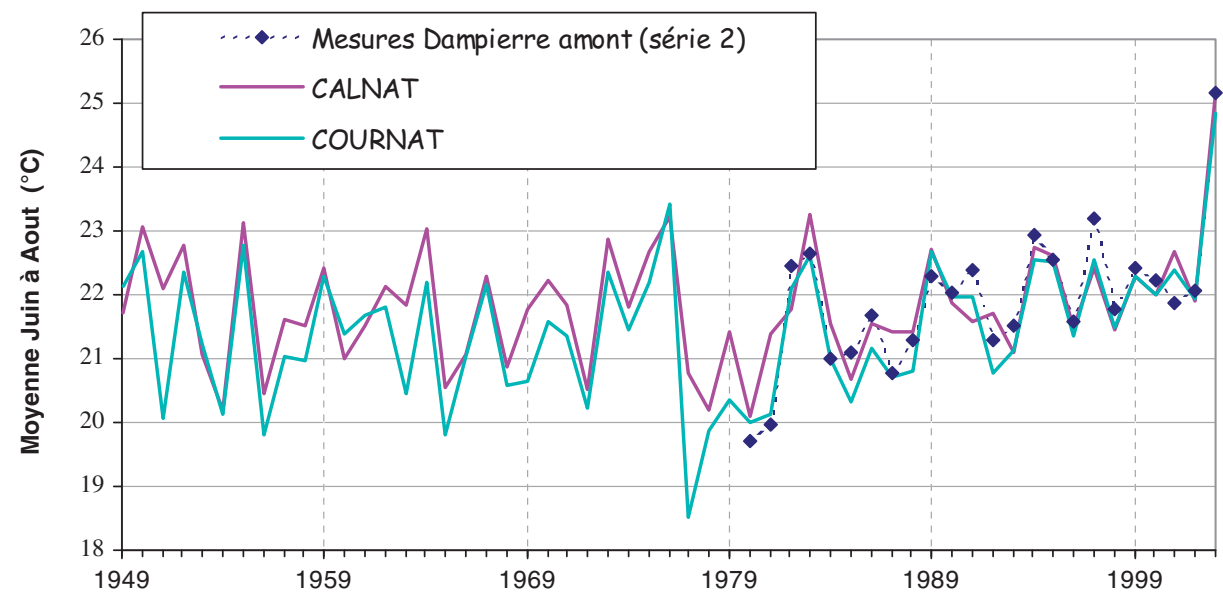

Fig. 12. Moyennes trimestrielles (juin-août) des températures d'eau calculées par CALNAT et COURNAT à partir des chroniques de mesures de quatre paramètres atmosphériques relevés en routine à la station Météo-France de Tours et (pour COURNAT) de mesures de débit de la Loire à Gien (années 1949-2003) ; et comparaison des résultats avec la série 2 de mesures de température de Loire au site Dampierre amont (années 1980-2003).

Fig. 12. Quarterly (June-August) averages of water temperatures calculated by CALNAT and COURNAT from data sets of 4 routine atmospheric parameters collected at the Météo-France station of Tours and from (for COURNAT) measurements of the Loire discharges at Gien (years 1949-2003); comparison with the Loire temperature data set (series 2) at Dampierre (years 1980-2003). 


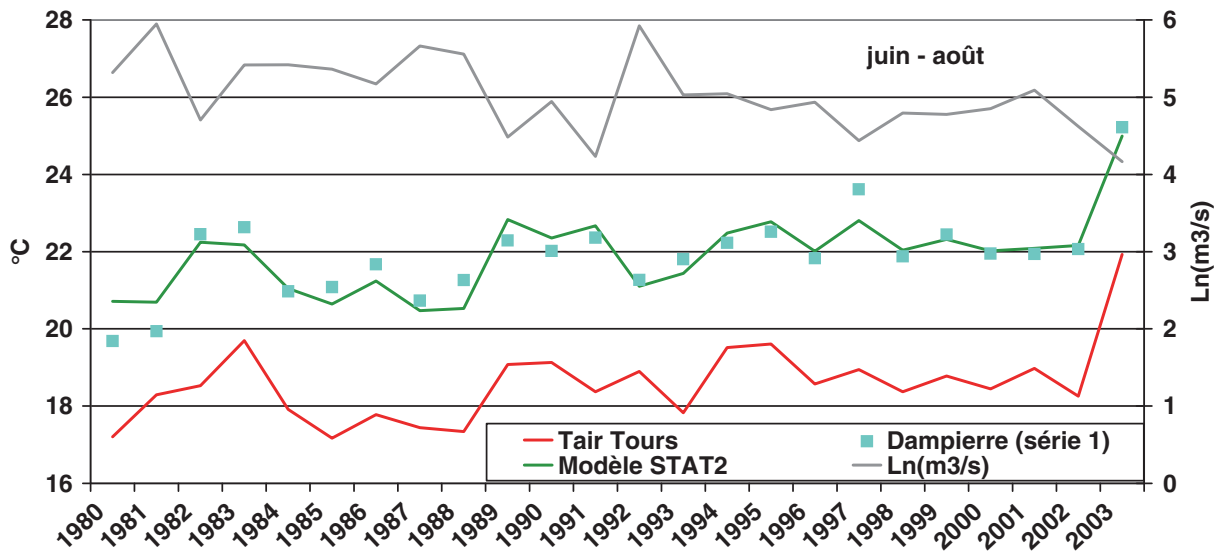

Fig. 13. Comparaison sur le trimestre $1^{\text {er }}$ juin- 31 août des moyennes des températures de Loire mesurées en continu à la station Dampierre amont (série 1), des températures d'air mesurées à la station Météo-France de Tours, du débit mesuré à Gien et des températures d'eau calculées par un modèle statistique de régression multilinéaire (STAT2) ayant la température trimestrielle d'air à Tours et le logarithme népérien du débit trimestriel à Gien comme variables explicatives (années 1980-2003).

Fig. 13. Comparison for the trimester 1st June-31 August of the average values of continuous measurements of mid-Loire temperature at the Dampierre amont station (series 1), air temperature measurements at the Météo-France station of Tours, Loire discharge measurements at Gien and water temperature calculated by a multilinear regression statistical model (STAT2) taking the quarterly air temperature at Tours and the Neperian logarithm of the quarterly Loire discharge at Gien as explaining variables (years 1980-2003).

à l'évolution du débit, si l'on considère l'équation du modèle. Ainsi la tendance à l'échauffement de l'eau est expliquée dans STAT2 à part égale par la température d'air $(51 \%)$ et par le débit ${ }^{(11)}(49 \%)$.

Le poids donné au débit comparativement aux données atmosphériques locales dans l'explication de

(11) On notera que la part du débit dans l'explication de la tendance d'échauffement de l'eau apparaît légèrement renforcée dans STAT2 par rapport à STAT1 (Fig. 14) qui donne une répartition $66-34 \%$ pour les influences respectives de la température d'air et du débit sur la période 1976-2003. l'échauffement de la Loire sur 19802003 est là encore plus élevé avec le modèle statistique multilinéaire STAT2 qu'avec ce qu'indiquent les modèles CALNAT-COURNAT à base physique.

\subsection{Analyse de tendance des températures estivales de la Loire moyenne sur la période 1949-2003}

En appliquant CALNAT et COURNAT sur la période 1949-2003, on trouve une tendance à l'échauffement moyen de l'eau de $0,3^{\circ} \mathrm{C}$ et $0,7^{\circ} \mathrm{C}$ sur ces 55 ans pour le trimestre 15 juin15 septembre (Tab. IV et Fig. 15) : cela donne un taux d'accroissement 


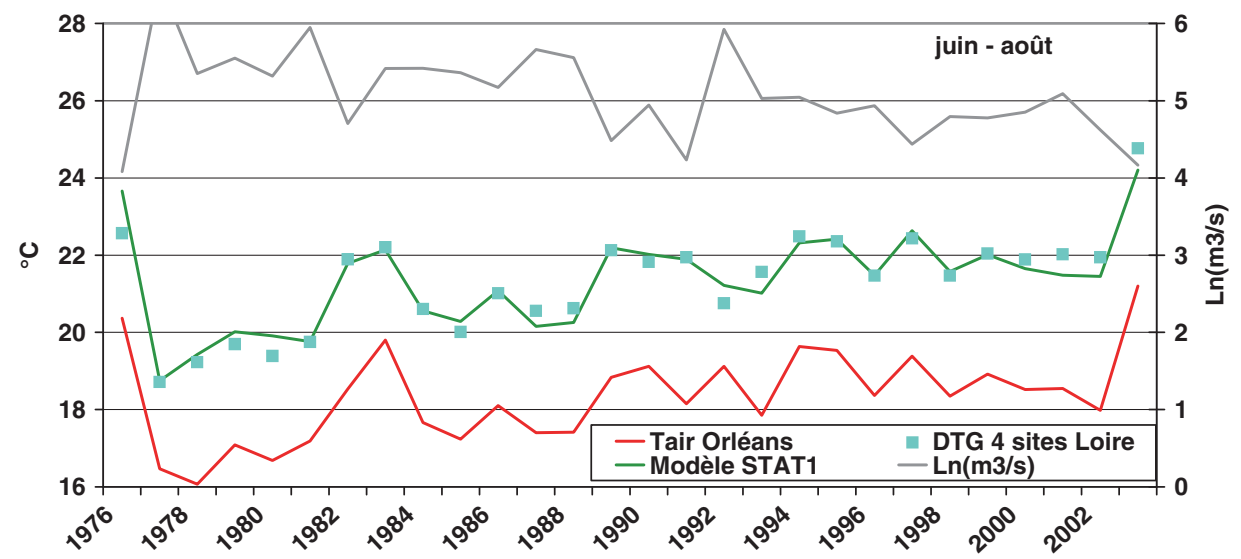

Fig. 14. Comparaison sur le trimestre $1^{\text {er }}$ juin- 31 août des moyennes des températures de Loire mesurées en continu à 4 stations de Loire moyenne (Belleville, Dampierre, Saint-Laurent et Avoine), des températures d'air mesurées à la station Météo-France d'Orléans, du débit mesuré à Gien et des températures d'eau calculées par un modèle statistique de régression multilinéaire (STAT1) ayant la température trimestrielle d'air à Orléans et le logarithme népérien du débit trimestriel à Gien comme variables explicatives (années 1976-2003).

Fig. 14. Comparison for the trimester 1st June-31 August of the average values of (1) continuous measurements of mid-Loire temperature at 4 stations (Belleville, Dampierre, Saint-Laurent et Avoine): (2) air temperature measurements at the Méteo France station of Orléans; (3) Loire discharge measurements at Gien; (4) water temperature calculated by a multilinear regression statistical model (STAT1) taking the quarterly air temperature at Orléans and the Neperian logarithm of the quarterly Loire discharge at Gien as explaining variables (years 1976-2003).

Tableau IV. Test de régression linéaire appliqué sur la période 1949-2003 aux moyennes trimestrielles 15 juin- 15 septembre de six paramètres mesurés sur la période et de résultats de deux modèles de température d'eau : CALNAT et COURNAT.

Table IV. Linear regression test applied to quarterly (15 June-15 September) averages of (1) six measured parameters ; (2) results of two water temperature models: CALNAT and COURNAT (years 1949-2003).

\begin{tabular}{|c|c|c|c|c|c|}
\hline Météo et Hydrologie & Unité & Variation (unité/(1949-2003)) & IC Binf. $90 \%$ & IC Bsup. $90 \%$ & Significativité \\
\hline Température d'air Tours & ${ }^{\circ} \mathrm{C}$ & 0,90 & 0,05 & 1,76 & $91 \%$ \\
\hline Pression de vapeur Tours & $\mathrm{hPa}$ & -0.54 & $-1,31$ & 0,22 & $75 \%$ \\
\hline Vitesse de vent $10 \mathrm{~m}$ Tours & $\mathrm{m} / \mathrm{s}$ & 0.39 & 0,16 & 0.61 & $99 \%$ \\
\hline Nébulosité Tours & octa & -0.42 & -0.86 & 0.02 & $88 \%$ \\
\hline Débit Gien & $\mathrm{m} 3 / \mathrm{s}$ & -16.8 & -89 & 6i) & $29 \%$ \\
\hline Température d'eau & Unité & Variation $\left({ }^{\circ} \mathrm{C} /(1949-2003)\right)$ & IC Binf. $90 \%$ & IC Bsup. $90 \%$ & Significativité \\
\hline CALNAT & ${ }^{\circ} \mathrm{C}$ & 0.30 & $-(0,40)$ & 7,90 & $52 \%$ \\
\hline COURNAT & ${ }^{\circ} \mathrm{C}$ & 0,70 & $-0,06$ & 1,46 & $86 \%$ \\
\hline
\end{tabular}

annuel au moins 5 fois plus faible que sur la période 1980-2003. On note de surcroît que ces évolutions sont statistiquement moins significatives (52 \% selon CALNAT et $86 \%$ selon COURNAT).
L'échauffement modéré calculé par CALNAT comparativement à celui de 1980-2003 est attribuable à l'impact global faible des tendances de variations des quatre paramètres atmosphériques qui cette fois ne jouent 


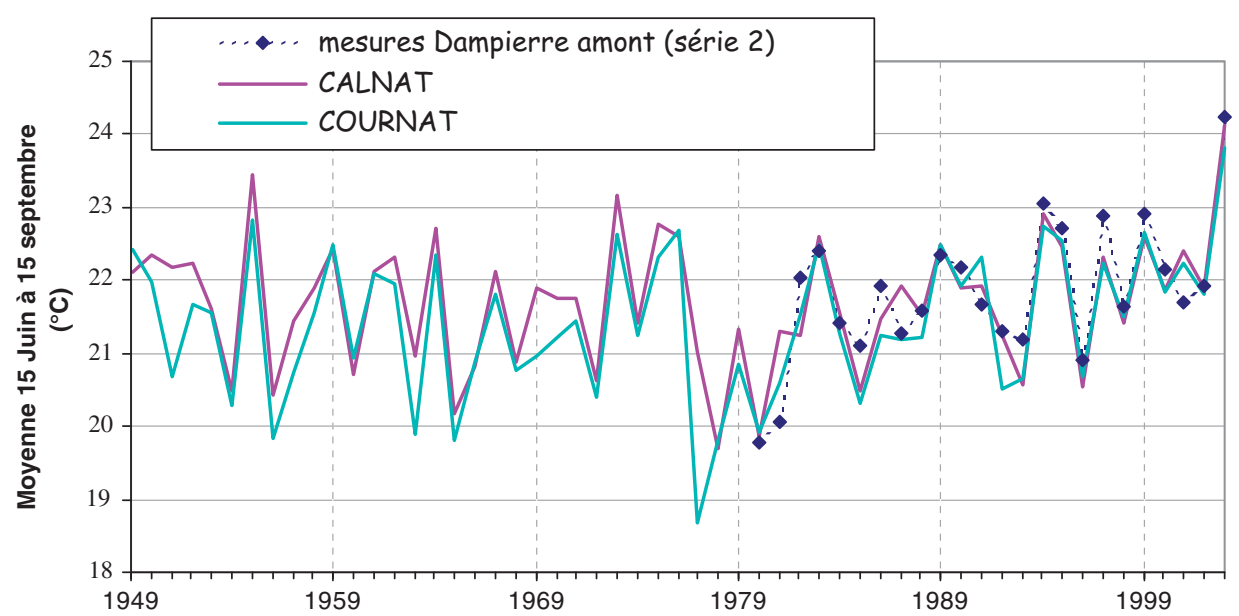

Fig. 15. Moyennes trimestrielles 15 juin-15 septembre des températures d'eau calculées par CALNAT et COURNAT à partir des chroniques de mesures de quatre paramètres atmosphériques relevés en routine à la station Météo-France de Tours et (pour COURNAT) de mesures de débit de la Loire à Gien (années 1949-2003) ; et comparaison avec la série 2 de mesures de température de Loire à Dampierre (années 1980-2003).

Fig. 15. Quarterly averages (15 June-15 September) of water temperatures calculated by CALNAT and COURNAT from data sets of 4 routine atmospheric parameters measured at the Météo-France station of Tours and (for COURNAT) of the Loire discharge at Gien (1949-2003) ; comparison with the data set (series 2) of Loire temperature at Dampierre (years 1980-2003).

plus toutes dans le même sens et se neutralisent : sur le même principe que celui retenu pour la période 1980-2003, on peut en effet évaluer que les tendances de variation ${ }^{(12)}$ en moyenne trimestrielle sur la période 1949-2003 (Tab. IV et Fig. 16) de la température d'air $\left(+0,9^{\circ} \mathrm{C}\right)$, de la pression de vapeur d'eau $(-0,55 \mathrm{hPa})$, de

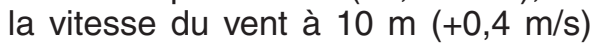
et de la nébulosité ( $-0,4$ octa) modifient la température naturelle locale $\mathrm{T}_{\text {nat }}$ de respectivement $+0,4^{\circ} \mathrm{C}$, $-0,2^{\circ} \mathrm{C}, \quad-0,4^{\circ} \mathrm{C}$ et $+0,3^{\circ} \mathrm{C}$. Cela donne un échauffement cumulatif de

(12) Les variations pour les variables météorologiques température de l'air et vitesse de vent sont significatives à plus de 90 \% .
$0,1{ }^{\circ} \mathrm{C}$ à comparer à la tendance d'échauffement de $0,3^{\circ} \mathrm{C}$ donnée par CALNAT. La différence de $0,2^{\circ} \mathrm{C}$ peut être liée à la répartition dissociée et uniforme à chaque pas tri-horaire de valeurs constantes de variation de paramètre atmosphérique caractérisant une tendance à l'échelle trimestrielle. On note par exemple que la tendance d'augmentation de la température d'air à Tours en moyenne trimestrielle sur 1949-2003 est de 0,5 ${ }^{\circ} \mathrm{C}$ sur une moitié de journée incluant la période nocturne et de $1,3^{\circ} \mathrm{C}$ sur l'autre moitié.

On constate ainsi que la tendance d'échauffement moyen de l'air en été sur la période 1980-2003 pour le trimestre 15 juin-15 septembre est de même intensité que la 
a

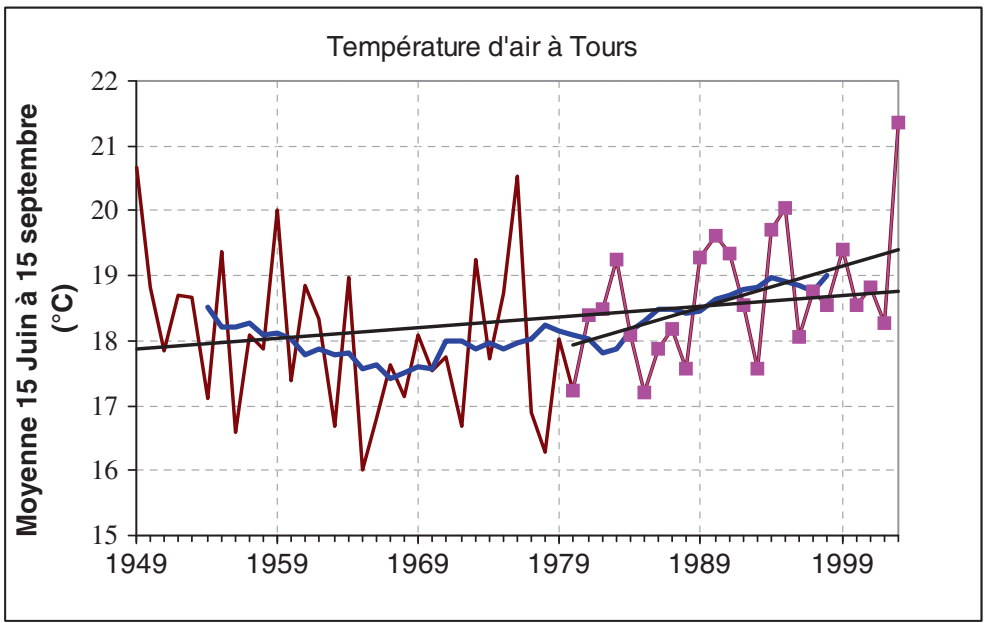

b

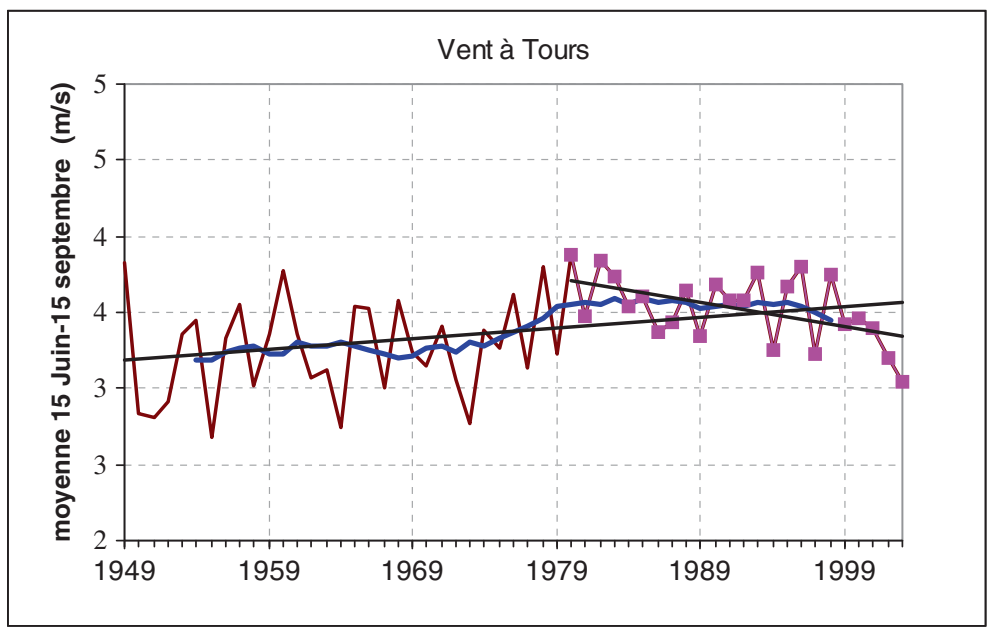

Fig. 16. Tendance d'évolution des moyennes trimestrielles (15 juin-15 septembre) de température d'air et de vitesse de vent à 10 mètres relevés à la station Météo-France de Tours sur les périodes 1949-2003 et 1980-2003. La courbe lissée représente des moyennes sur 11 années.

Fig. 16. Evolution trend of the quarterly (15 June-15 September) averages of air temperature and wind velocity at $10 \mathrm{~m}$ recorded at the Météo-France station of Tours (years 1949-2003 and 1980-2003). The smooth curve gives averages over 11 years. 
tendance d'augmentation de la température naturelle locale d'un plan d'eau en équilibre avec les conditions météorologiques de Tours, mais plus de deux fois supérieure sur la période 1949-2003. Ces résultats suggèrent qu'une relation trouvée entre variation de températures d'air et de plan d'eau n'est pas nécessairement extrapolable sur un plus grand nombre décennies.

Cela montre une nouvelle fois l'importance de la prise en compte des 4 paramètres atmosphériques dans le bilan thermique de la rivière. L'évolution de la vitesse de vent est particulièrement intéressante car son augmentation tendancielle évaluée sur les 55 ans pour la période 15 juin-15 septembre est de même niveau mais de sens opposé à la baisse tendancielle de 1980-2003, dont on a vu la contribution importante dans la tendance d'échauffement calculée par CALNAT sur ces 24 ans. L'évolution du vent en été aurait ainsi eu sur les 55 ans un effet refroidissant de la Loire moyenne - donc particulièrement marqué sur la période 1949-1980 - malgré un effet réchauffant significatif sur 1980-2003.

Ayant une formulation de la fonction de vent moins sensible que CALNAT à la variation de la vitesse du vent à Tours, COURNAT prend moins en compte l'effet refroidissant du vent. Cela peut expliquer que COURNAT donne une tendance d'échauffement un peu plus élevée de quelques dixièmes de ${ }^{\circ} \mathrm{C}$ que CALNAT sur 55 ans. En effet, dans le même temps (Tab. IV et Fig. 9), il n'y pas de tendance marquée sur le débit en niveau moyen et en fréquence d'apparition de hauts débits $\left(>300 \mathrm{~m}^{3} / \mathrm{s}\right)$. On notera par ailleurs que la pression de vapeur d'eau a un comportement similaire à la vitesse de vent : sa baisse tendancielle évaluée sur les 55 ans $(-0,55 \mathrm{hPa})$ est d'un niveau comparable mais de sens opposé à l'évolution tendancielle sur 1980-2003 $(+0,65 \mathrm{hPa})$.

\section{3 Étés chauds et froids pour l'écosystème Loire moyenne sur la période 1949-2003}

Si les tendances d'échauffement de l'eau sur 55 ans sont modérées en été pour la période 1949-2003, les résultats de CALNAT et COURNAT montrent (Figs. 12 et 15) une grande variabilité interannuelle que l'on peut par exemple apprécier en dégageant les étés les plus chauds et les plus froids pour l'écosystème Loire moyenne, le critère retenu étant la valeur la plus élevée des moyennes des températures d'eau trouvée chaque année sur 90,30 ou 7 jours consécutifs, notées $T_{\max 90}, T_{\max 30}$ et $T_{\max 7}$. Ces grandeurs ont la signification d'une dose thermique maximale reçue par l'écosystème aquatique sur une durée plus ou moins longue. On notera une analogie entre ces doses thermiques et le critère du mois le plus chaud retenu dans la classification biotypologique de Verneaux (1977) pour les cours d'eau.

L'année 2003 apparaît avec les deux modèles (Figs. 17 et 19) comme l'année record pour la dose thermique maximale reçue par l'écosystème fluvial aussi bien sur 7 jours consécutifs que sur 90 jours consécutifs. Les valeurs calculées par CALNAT et COURNAT pour ces deux grandeurs $T_{\max 7}$ et $T_{\max 90}$ sont très proches de celles données par les deux capteurs de 


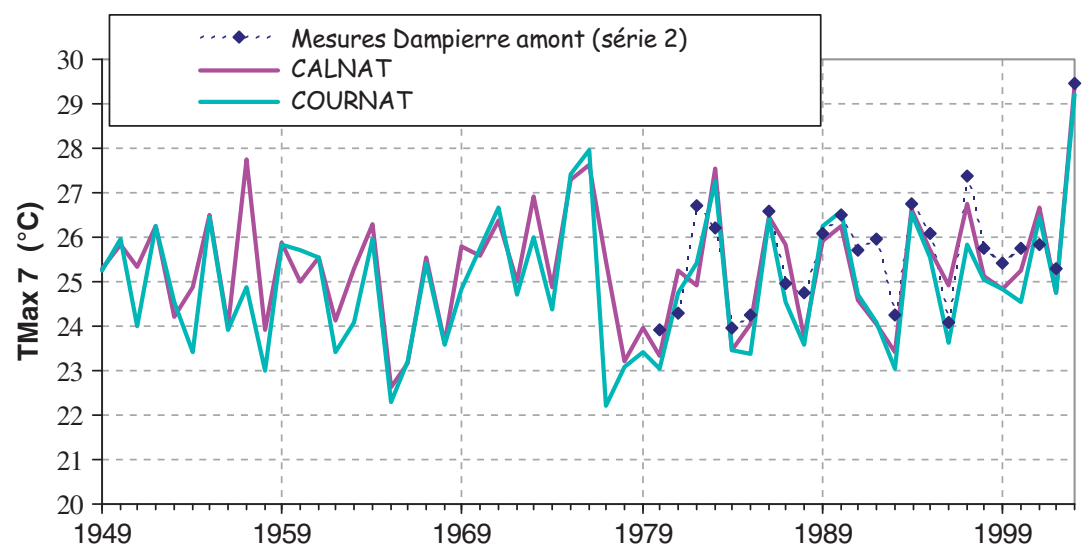

Fig. 17. Tracé de la moyenne des températures d'eau la plus élevée sur 7 jours consécutifs de l'année donnée par CALNAT et COURNAT; comparaison avec les résultats des mesures (série 2) de température de Loire à la station Dampierre amont (période 1949-2003).

Fig. 17. Highest average temperature for 7 consecutive days of the year given by CALNAT and COURNAT; comparison with observations (series 2) of the Loire temperature at Dampierre amont station (years 1949-2003).

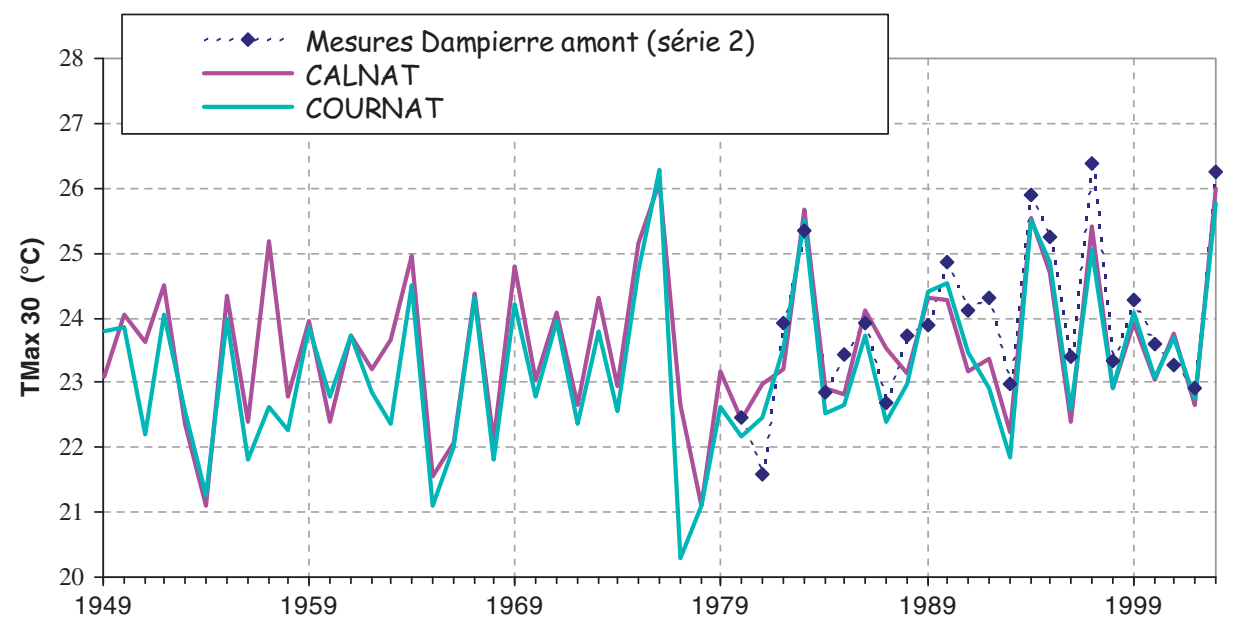

Fig. 18. Tracé de la moyenne des températures d'eau la plus élevée sur 30 jours consécutifs de l'année donnée par CALNAT et COURNAT; comparaison avec les résultats des mesures (série 2) de température de Loire à la station Dampierre amont (période 1949-2003).

Fig. 18. Highest average temperature for 30 consecutive days of the year given by CALNAT and COURNAT; comparison with observations (series 2) of the Loire temperature at Dampierre amont station (years 1949-2003). 


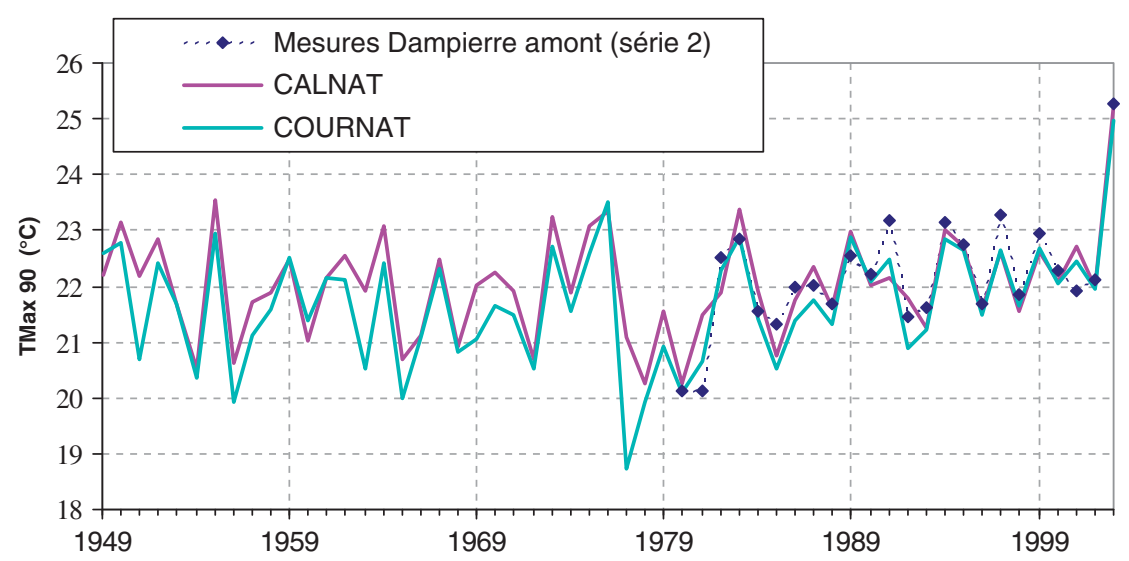

Fig. 19. Tracé de la moyenne des températures d'eau la plus élevée sur 90 jours consécutifs de l'année donnée par CALNAT et COURNAT; comparaison avec les résultats des mesures (série 2) de température de Loire à la station Dampierre amont (période 1949-2003).

Fig. 19. Highest average temperature for 90 consecutive days of the year given by CALNAT and COURNAT; comparison with observations (series 2) of the Loire temperature at Dampierre amont station (years 1949-2003).

mesure de température à la station Dampierre amont :

- T $\max 7$ calculée de $29,4^{\circ} \mathrm{C}$ avec CALNAT et de $29,2^{\circ} \mathrm{C}$ avec COURNAT,

- T $\max 7$ observée de $29,5^{\circ} \mathrm{C}$ avec les séries 1 et 2 de mesures Dampierre amont,

- Tmax90 calculée de $25,2^{\circ} \mathrm{C}$ avec CALNAT et de $25,0^{\circ} \mathrm{C}$ avec COURNAT,

- Tmax9o observée de $25,35^{\circ} \mathrm{C}$ et $25,25^{\circ} \mathrm{C}$ avec les séries 1 et 2 de mesures Dampierre amont.

Le caractère exceptionnel de l'année 2003 apparaît dans l'écart thermique avec la deuxième année la plus chaude de la série : $+1,5^{\circ} \mathrm{C}$ pour $\mathrm{T}_{\max 7}$ et $+2^{\circ} \mathrm{C}$ pour $\mathrm{T}_{\max 90}$.

Pour la dose thermique maximale reçue sur 30 jours consécutifs (Fig. 18), CALNAT met l'année 2003 en tête avec une $T_{\max 30}$ de $26,0^{\circ} \mathrm{C}$ confirmée à $0,3^{\circ} \mathrm{C}$ près par les mesures de Dampierre $\left(26,3{ }^{\circ} \mathrm{C}\right.$ et $26,25^{\circ} \mathrm{C}$ pour les séries 1 et 2 de mesures). Mais plusieurs années font jeu égal (année 1976(13) ou présentent des valeurs s'écartant de

(13) La température de $26,1^{\circ} \mathrm{C}$ calculée par CALNAT pour la dose thermique maximale reçue sur 30 jours consécutifs par l'écosystème fluvial en 1976 apparaît crédible, les mesures de température de Loire disponibles à SaintLaurent-des-Eaux amont cette année donnant $23,6^{\circ} \mathrm{C}$ : cela fait un écart de $2,5^{\circ} \mathrm{C}$ entre CALNAT et les données de Saint-Laurent, ce qui est assez proche de l'écart de $1,7^{\circ} \mathrm{C}$ que l'on trouve en 2003 entre les données de Saint-Laurent $\left(24,3^{\circ} \mathrm{C}\right)$ et les résultats de CALNAT $\left(26,0^{\circ} \mathrm{C}\right)$ dans des conditions hydrologiques proches. En 2003, on a un écart comparable de $2,0{ }^{\circ} \mathrm{C}$ entre les données de Saint-Laurent et celles de Dampierre $\left(26,3^{\circ} \mathrm{C}\right)$. On rappelle que Moatar et Gailhard (2006) attribuent aux apports de nappe les températures plus basses relevées à Saint-Laurent-des-Eaux comparativement à Dampierre. 
moins de $1^{\circ} \mathrm{C}(1983,1994,1997)$, ce que confirment les mesures.

Les mesures à Dampierre placent l'année $1997\left(26,3^{\circ} \mathrm{C}\right)$ au niveau de 2003, ce que ne confirme pas les stations de Belleville (qui donne une valeur plus basse de $2,2^{\circ} \mathrm{C}$ à celle de Dampierre en 1997, ce qui est un écart très élevé comparativement à celui des autres années) et celles de Saint-Laurent et Avoine qui la placent comme CALNAT derrière 2003.

On notera qu'il est tout à fait remarquable que CALNAT calé sur la base d'une expertise antérieure à l'année de la canicule, ait une telle performance pour 2003, les valeurs de $T_{\max 7}, T_{\max 30}$ et $T_{\max 90}$ s'écartant d'au plus $0,3^{\circ} \mathrm{C}$ de celles obtenues avec les capteurs de mesure à l'amont de la centrale de Dampierre.

$\mathrm{Ce}$ sont ainsi uniquement les conditions extrêmes de température d'air - vent - nébulosité - humidité qui expliquent les trois pics thermiques record de la Loire en 2003 aux échelles de temps considérées et pas le niveau très bas alors observé pour le débit du fleuve (minimum de $50 \mathrm{~m}^{3} / \mathrm{s}$ à Gien) : la seule influence du débit dans les évolutions calculées est son niveau durable en dessous de $300 \mathrm{~m}^{3} / \mathrm{s}$ - cas courant en été - permettant à la rivière de ne pas subir d'influence climatique du bassin versant amont. Dans de telles conditions, le principal impact d'un bas débit est d'augmenter l'amplitude jour-nuit de la température de l'eau, les moyennes hebdomadaires ou mensuelles n'étant pas affectées.

Au bas du classement, COURNAT - plus adapté que CALNAT pour un débit fluvial dépassant durablement $400 \mathrm{~m}^{3} / \mathrm{s}$ - place l'été 1977 comme le plus froid de la période 1949-2003 avec des valeurs de $\mathrm{T}_{\max 7}, \mathrm{~T}_{\max 30}$ et $\mathrm{T}_{\max 90}$ de $22^{\circ} \mathrm{C}, 20^{\circ} \mathrm{C}$ et $19^{\circ} \mathrm{C}$ respectivement. Ces niveaux sont confirmés cette année là par les températures mesurées à Belleville et en aval d'Orléans.

Les écarts importants de dose thermique maximale entre les étés froids et les étés chauds sont une traduction de la forte variabilité interannuelle coexistant avec la tendance multi-décennale à l'échauffement de la Loire. II n'est pas rare par exemple de trouver un écart de $3^{\circ} \mathrm{C}$ entre deux années consécutives pour $T_{\max 30}$, l'écart maximal étant de $6{ }^{\circ} \mathrm{C}$ entre les années 1976 et 1977. Ce record 19761977 apparaît difficile à battre, les deux années étant classées au premier rang et au dernier rang de l'ensemble des 55 ans pour le critère thermique considéré.

Ces résultats sont cohérents avec ceux de Moatar et Gailhard (2006) qui dégagent aussi les deux années extrêmes 2003 et 1977 en moyenne sur le trimestre juin - août pour la période 1949-2003 mais également pour la période 1881-2003. On le confirme en appliquant le modèle statistique STAT2 - de même type que celui de Moatar et Gailhard - à toute la période 1949-2003 (Fig. 20). Toutefois, en donnant plus de poids au débit que les modèles à base physique CALNAT/COURNAT dans l'explication des températures de la Loire, cette approche statistique accentue l'échauffement de l'eau à l'étiage, ce qui apparaît nettement pour les étés secs 1949, 1952 et 1976.

\subsection{Pertinence des modèles en période d'étiage}

Compte tenu de la faible réponse de la profondeur d'inertie thermique aux échelles de temps considérées et de 


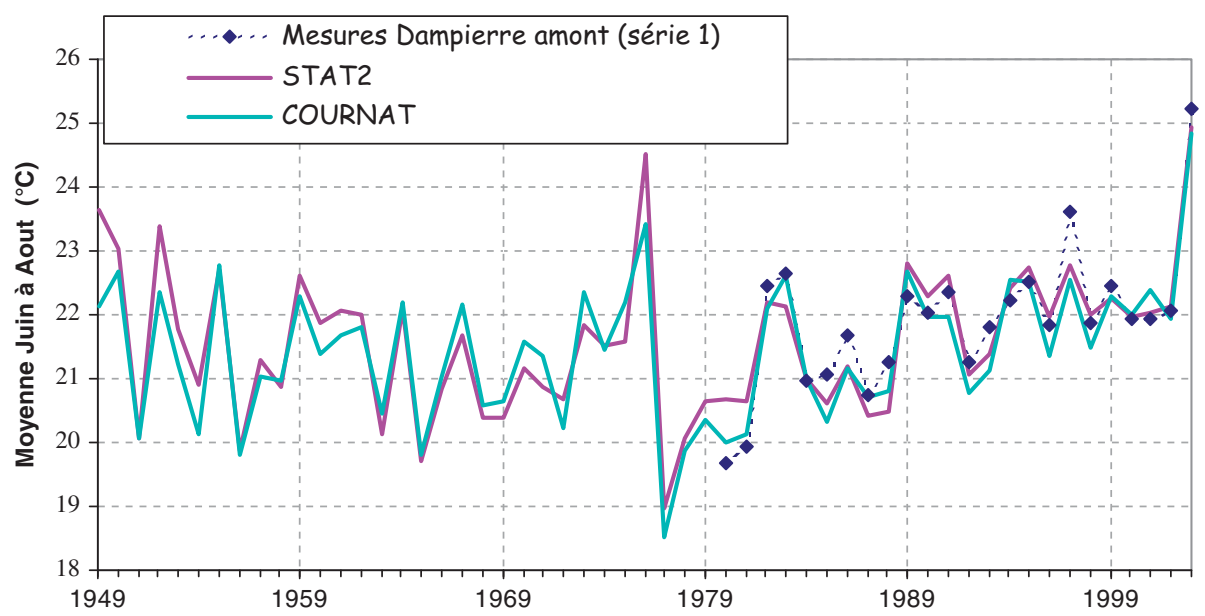

Fig. 20. Moyennes trimestrielles (juin-août) des températures d'eau calculées par COURNAT et STAT2 (années 1949-2003) ; et comparaison des résultats avec la série 1 de mesures de température de Loire au site Dampierre amont (années 1980-2003).

Fig. 20. Quarterly (June-August) averages of water temperatures calculated by COURNAT and STAT2 (years 1949-2003); comparison with the Loire temperature data set (series 1) at Dampierre (years 1980-2003).

la très bonne performance de CALNAT et COURNAT pour reproduire les caractéristiques thermiques de la Loire pendant l'étiage 2003 marqué par des débits durablement voisins de $50 \mathrm{~m}^{3} / \mathrm{s}$, on peut considérer que les résultats des deux modèles sont peu entachés d'erreurs pour des débits moyens deux fois inférieurs à ce niveau, en se limitant au secteur amont d'Orléans peu alimenté par la nappe dans de telles conditions.

Des doutes sur la pertinence des résultats des deux modèles ne se poseraient alors que pour l'année 1949 qui est la seule année de la période étudiée pour laquelle un débit moyen journalier inférieur à $25 \mathrm{~m}^{3} / \mathrm{s}$ a été enregistré à Gien sur la période 19492003 :

- plus bas débit sur une journée (le 8 août et 5 jours de la période $18-23$ août) : $11 \mathrm{~m}^{3} / \mathrm{s}$,
- plus bas débit moyen sur 7 jours consécutifs (dans l'intervalle 1624 août) : $12 \mathrm{~m}^{3} / \mathrm{s}$,

- plus bas débit moyen sur 30 jours consécutifs (dans l'intervalle $1^{\mathrm{er}}$ août-3 septembre) : $14 \mathrm{~m}^{3} / \mathrm{s}$,

- plus bas débit moyen sur 90 jours consécutifs (dans l'intervalle 12 juillet-5 novembre) : $20 \mathrm{~m}^{3} / \mathrm{s}$.

Comme le fleuve s'est asséché cette année-là à Orléans (Moreau, 2004), le domaine de représentativité des modèles couvre nécessairement un domaine spatial plus restreint que les autres années, le tronçon GienDampierre y étant certainement inclus. En effet, les principales pertes d'eaux de Loire par infiltration se produisent au-delà de Dampierre. Dans ce domaine de représentativité, il est peu probable que les records de température d'eau de 2003 aient été battus, dans la mesure où les valeurs de 
$T_{\max 7}, T_{\max 30}$ et $T_{\max 90}$ calculées par CALNAT pour 1949 sont inférieures de $4^{\circ} \mathrm{C}$ et $2^{\circ} \mathrm{C}$ à celles de 2003 . Par contre, en fonction de l'étalement de l'écoulement et à l'approche du secteur asséché, on a pu se trouver dans des conditions d'écoulement à très faible profondeur assimilable à une masse d'eau sans inertie thermique dans les secteurs sans apport de nappe, et donc avec une amplitude jour-nuit importante conduisant à des valeurs élevées de température autour du midi solaire.

\section{CONCLUSION}

Plusieurs points peuvent être dégagés de l'application des modèles déterministes à base physique CALNAT et COURNAT à la Loire moyenne et de la comparaison des résultats obtenus avec les chroniques de mesures en continu de température d'eau et avec ceux donnés par un modèle purement statistique reprenant l'approche de Moatar et Gailhard (2006).

En premier lieu, le modèle CALNAT de température naturelle locale reproduit exactement (à moins de $0,3^{\circ} \mathrm{C}$ près) avec un jeu de paramètres standard la valeur la plus élevée des moyennes des températures de Loire constatées en 2003 à Dampierre sur 90,30 ou 7 jours consécutifs. Les seules entrées variables du modèle sont les valeurs tri-horaires de 4 paramètres atmosphériques relevés en routine à la station MétéoFrance de Tours : température d'air, pression de vapeur d'eau, vitesse du vent à 10 mètres et nébulosité du ciel. Ce sont ainsi uniquement les conditions extrêmes de ces quatre paramètres qui expliquent les pics thermiques record de la Loire en 2003 aux échelles de temps considérées et non le très faible débit fluvial (jusqu'à $50 \mathrm{~m}^{3} / \mathrm{s}$ à Gien) alors observé.

On notera également l'aptitude de COURNAT à reproduire l'impact refroidissant des conditions de haut débit qui sortent du domaine de validité de CALNAT; ce qui peut expliquer que COURNAT a une bien meilleure performance que CALNAT pour reproduire la tendance d'échauffement de la Loire moyenne sur le trimestre juinaoût de 1980 à 2003.

En retenant comme critères les valeurs les plus élevées des moyennes des températures de Loire sur 90, 30 ou 7 jours consécutifs, on trouve avec CALNAT et COURNAT que 2003 est l'année de la période 19492003 durant laquelle la Loire à Dampierre a été la plus chaude, quelques années, en particulier 1976, faisant jeu ou presque jeu égal à l'échelle de 30 jours. COURNAT signale l'année 1977 comme la plus froide des 55 années pour les trois critères considérés. Ces résultats sont cohérents avec ceux de Moatar et Gailhard (2006) qui identifient aussi ces deux années extrêmes sur le trimestre juin-août pour 1949-2003 et pour la période plus longue 1881-2003.

Un autre élément remarquable de l'application de la version standard de CALNAT qui ne prend pas en compte les variations de débit et de COURNAT qui les considère est la constatation de la capacité des ces outils à reproduire respectivement $84 \%$ et $92 \%$ de la tendance d'échauffement de la Loire constatée à la station de mesures de Dampierre $\left(1,85^{\circ} \mathrm{C}\right)$ de 1980 à 2003 sur le trimestre 15 juin-15 septembre. Ceci met en relief l'effet prépondérant des 
variations des conditions atmosphériques dans l'explication de l'échauffement de la Loire moyenne sur la période.

On a constaté également que les quatre paramètres atmosphériques relevés à Tours avaient chacun un poids sur les tendances d'évolution de la température naturelle locale en été aussi bien sur 1980-2003 que sur 1949-2003, avec un effet convergent sur 1980-2003 mais antagoniste sur 1949-2003. On note dans ces évolutions que la température d'air représente le facteur le plus influent, mais que l'effet des autres paramètres, notamment du vent, peut être d'intensité assez proche.

Ces résultats conduisent à recommander de considérer les quatre paramètres météorologiques pour caractériser les évolutions de la température de rivière et de bien schématiser la vitesse du vent au dessus de l'écoulement. Ils montrent aussi l'intérêt de pouvoir disposer de modèles de prévision de changement climatique ayant une qualité de pronostic de la vitesse de vent, de l'humidité de l'air et du rayonnement solaire de même niveau que pour la température d'air. Enfin, ils posent (Hendrickx et Sauquet, 2008) la question de la robustesse d'approches courantes ne retenant comme paramètre atmosphérique que le paramètre température de l'air pour prévoir les températures futures de masses d'eau sous l'effet du changement climatique.

L'approche purement statistique de Moatar et Gailhard (2006) qui repose sur une équation linéaire retenant la moyenne des températures d'air à Orléans ou Tours et le logarithme népérien de la moyenne des débits du fleuve à Gien comme variables explicatives, représente les températures moyennes estivales de la Loire moyenne sur la période 1980-2003 avec une précision comparable à celle de CALNAT et COURNAT. II apparaît cependant que l'approche déterministe à base physique de CALNAT et COURNAT est plus adaptée pour analyser des tendances d'évolution à long terme, la corrélation existante en été entre débit et température d'air conduisant le modèle statistique à donner un poids excessif au débit dans la tendance à l'échauffement de la Loire en été sur les 24 ans.

Plus généralement, et en lien avec des études qui cherchent à explorer le futur hydro-climatique, on peut faire le constat que nos modélisations, qu'elles soient hydrologiques ou comme ici thermiques, sont souvent calées et validées sur des critères de type moyenne (moindre carré, Nash, ...). Ces critères expriment toutefois leur limite en ne permettant pas de traduire l'aptitude d'un modèle à savoir représenter une fluctuation de faible intensité et de basse fréquence. Aussi, l'exercice de validation des modèles sur les grandes fluctuations pluriannuelles, comme ici sur les tendances, nous semble être utile pour être plus confiant dans l'aptitude de ces modèles à se projeter dans un nouveau contexte hydroclimatique.

La démarche effectuée ici mériterait d'être poursuivie en intégrant les données de stations météorologiques voisines et en examinant ces tendances sur des pas de temps plus courts depuis le mois jusqu'à l'échelle de la journée, pas de temps sur lesquels la qualité descriptive d'outils comme CALNAT/COURNAT est bonne. On peut en effet s'attendre avec ces nouvelles conditions 
à trouver une pondération différente des liens mis en évidence ici entre paramètres hydrométéorologiques et températures d'eau.

\section{Addendum}

Les résultats de CALNAT et COURNAT relatifs aux valeurs les plus élevées des moyennes des températures de Loire sur 30 et 90 jours consécutifs de l'année (période 1949-2003) et leur comparaison avec des mesures de températures de la Loire moyenne ont été présentés lors d'un exposé au colloque Hydroécologie 2004 (Gailhard et al., 2004).

Les auteurs remercient Françoise Siclet pour ses suggestions d'enrichissement du texte sur le plan statistique, Anne Dupeyrat pour sa participation au test statistique, ainsi que les relecteurs de l'article pour leurs remarques constructives.

\section{RÉFÉRENCES \\ BIBLIOGRAPHIQUES}

Dupeyrat A. \& Bertier C., 2006. Impact du changement climatique sur les régimes hydrologiques et thermiques de la Loire. Rapport EDF H-P76-200500904-FR, Électricité de France, Paris.

Gailhard J., Gosse P. \& Moatar F., 2004. Le palmarès des étés chauds et froids pour l'écosystème Loire moyenne sur les 55 dernières années. Communication au Colloque Hydroécologie, 21 et 22 octobre 2004. Clamart

Gilbert A. \& Roult D., 1986. Modélisation thermique des rivières. Notice d'utilisation des programmes de simulation. Rapport EDF HE31/87/3, Électricité de France, Paris.
Gosse P., 2005. Évaluation de l'échauffement de la Loire par la centrale de Dampierre (références hydrométéorologiques des années 1980 à 2003). Rapport EDF HP-76/04/012/A (référence EUREKA H-P76-2005-01396FR), Électricité de France, Paris.

Gosse Ph. \& Gailhard J., 1996. Température de la Garonne et du Tarn à l'étiage à proximité du réservoir de Malause : analyse de mesures collectées en 1995. Rapport ECOBAG/EDF HE-31/96/16 (référence EDF EUREKA H-E30-1996-02979-FR), Électricité de France, Paris.

Gosse Ph., Gras R., Royer T. \& Gailhard J., 1996. Effet d'un changement climatique sur la température d'un cours d'eau avec examen pour les rivières françaises d'un doublement de la concentration atmosphérique en $\mathrm{CO}_{2}$. Rapport EDF HE31/96/33 (références EDF EUREKA H-E30-1996-04745-FR, INIST-CNRS, 26165 C), Électricité de France, Paris.

Gosse Ph., Gailhard J., Gras R. \& Royer T., 1997. Températures naturelles de plans d'eau peu profonds par grandes régions de France. Rapport EDF-HE-31/97-29 (référence EDF EUREKA H-E30-1997-04611-FR). Électricité de France, Paris.

Gras R., 1969. Simulation du comportement thermique d'une rivière à partir des données fournies par un réseau classique d'observations météorologiques. IAHR Congress, Kyoto, 1969. Article A53, Vol. 1, pp. 491-502.

Gras R., 1970. Estimation des éléments du bilan radiatif pour le calcul des températures naturelles d'un secteur de cours d'eau. Rapport EDF HF/041/70/n²6, Électricité de France, Paris.

Gras R., Fleuret J. \& Gilbert A., 1971. Simulation du comportement thermique 
des cours d'eau : courbes d'ajustement du modèle mathématique à diverses rivières de France. Rapport EDF HF/041/71/n 49 , Électricité de France, Paris.

Gras R., Albignat J.P. \& Gosse Ph., 1983. Changes in the thermal behaviour of streams associated with the construction of hydraulic projects. Proceedings of the 20th IAHR Congress, (U.I.N.I.T.I.), 3-9 September 1983, Moscow. USSR, Vol. II, pp. 1218.

Gras R., Gilbert A. \& Roult D., 1986. Numerical computation of natural river temperatures. Proceedings of the international Conference on Water Quality modeling in Natural Environnement, Bournemouth, 10-13 juin 1986, pp. 457-472.

Hendrickx F. \& Sauquet E., 2008. Les constats et les prévisions d'évolution de l'hydrologie et de la température des cours d'eau. Communication au Colloque Hydroécologie, 16 et 17 octobre 2008, Clamart, http ://rd.edf.com/ fichiers/fckeditor/File/2_2-Sauquet \%20 Hendrickx.pdf.

Jacquet J., 1983. Simulation of thermal regimes of rivers, Mathematical modelling of water quality, G.T. Orlob (Ed.), International Series on applied systems analysis, Vol. 12. pp. 150-175. J. Wiley and sons, Chichester, 1983.

Klein P. \& Momal D., 1979. Méthode d'estimation du rayonnement solaire. Sa mise en œuvre numérique. Rapport EDF HE31/79/15, Électricité de France, Paris.

Luck M., 2004. Système MASCARET : Note de principe de l'outil TRACER (transport de traceurs) et des modules de qualité d'eau $\mathrm{O}_{2}$, BIOMASS, EUTRO, MICROPOL et THERMIC. Rapport EDF HP-75/03/047/A (référence EDF EUREKA H-P75-200402678-FR), Électricité de France, Paris.

Moatar F. \& Gailhard J., 2006. Water temperature behaviour in the River Loire since 1976 and 1881. Comptes Rendus Geosciences 338 (5) : 319-328.

Moreau F., 2004. Gestion des étiages sévères : l'exemple de la Loire. Houille Blanche (4) : 70-76.

Poulin M., 1980. Modélisation du comportement thermique des cours d'eau. Application au Rhin. Thèse docteuringénieur, Université Paris-VI.

Renard B., 2006. Détection et prise en compte d'éventuels impacts du changement climatique sur les extrêmes hydrologiques en France. Thèse INP, Grenoble.

Salençon M.J. \& Thebault J.M., 1997. Modélisation d'écosystème lacustres. Application à la retenue de Pareloup (Aveyron). Masson, Paris, 1997, $184 \mathrm{pp}$.

Siclet F., Gailhard J. \& Ciffroy P., 2001. Radionuclides concentrations in the Loire river system resulting from routine discharges of five nuclear power plants. Présentation au Congrès IPSN-ECORAD 2001, 3-8 septembre 2001.

Verneaux J., 1977. Biotypologie de l'écosystème " eaux courantes ». Détermination approchée de l'appartenance typologique d'un peuplement piscicole. CR Acad. Sc. Paris 284 : 675-678. 


\section{Annexe 1 : Description du modèle CALNAT}

II s'agit d'un rappel des équations déjà présentées dans des documents antérieurs (par exemple Salençon et Thébault, 1997; Luck, 2004); Gosse et Gailhard (1996) ont fourni le code FORTRAN du modèle.

CALNAT calcule l'évolution temporelle de la température spatiale moyenne d'une masse d'eau relativement homogène, sous l'effet des échanges d'énergie à l'interface eauatmosphère, l'effet thermique des entrées d'eau et des échanges d'énergie avec le fond étant supposé secondaire.

L'équation résolue à pas tri-horaire s'écrit :

$$
\begin{aligned}
\frac{\partial T}{\partial t}= & \frac{V}{C p . H}(R S+R A-R E \\
& -C V-C E)
\end{aligned}
$$

avec

RS : flux thermique surfacique dû au rayonnement solaire pénétrant $\left(\mathrm{W} . \mathrm{m}^{-2}\right)$;

RA : flux thermique surfacique dû au rayonnement atmosphérique (W. $\mathrm{m}^{-2}$ );

$R E$ : flux thermique surfacique dû au rayonnement du plan d'eau (W. $\mathrm{m}^{-2}$ );

CV : flux thermique surfacique dû à la convection (W. $\mathrm{m}^{-2}$ );

CE : flux thermique surfacique dû à l'évaporation (W.m $\left.{ }^{-2}\right)$;

$\mathrm{Cp}$ : chaleur massique de l'eau $\left(\mathrm{J} \cdot \mathrm{kg}^{-1} \cdot \mathrm{K}^{-1}\right)$;

$\mathrm{v}$ : volume massique de l'eau $\left(\mathrm{m}^{3} \cdot \mathrm{kg}^{-1}\right)$;
$\mathrm{H}$ : profondeur d'inertie thermique $(\mathrm{m})$.

RS est calculé à partir des mesures tri-horaires de nébulosité du ciel (neb exprimé en octas) par le code RAYSOL (Klein et Momal, 1979) qui intègre sur un pas de temps à fournir l'équation :

$R S=y(\sinh )^{z}\left(1-0,65(\text { neb } / 8)^{2}\right)(1-A)$

avec

neb mesure de nébulosité à la station météorologique considérée (en octas);

h est la hauteur angulaire (en radian) qui est calculée et dépend du jour de l'année, de l'heure de la journée et des coordonnées géographiques de la station;

$y$ et $z$ sont les constantes permettant de caractériser l'influence du degré de pureté du ciel, 3 couples $(\mathrm{y}, \mathrm{z})$ de base étant proposés dans RAYSOL pour caractériser un ciel très pur, moyennement pur ou typique d'une zone industrielle. L'option ciel moyennement pur est retenue ici, ce qui donne $y=1080 \mathrm{~W} \cdot \mathrm{m}^{-2}$ et $\mathrm{z}=1,22$;

A est l'albédo du plan d'eau donné par une table fournie par le code RAYSOL.

RA est calculé par l'équation :

$$
\begin{aligned}
R A= & e_{\text {air }} \sigma\left(T_{\text {air }}+273,15\right)^{4} \\
& \times\left(1+k\left(\frac{n e b}{8}\right)^{2}\right)
\end{aligned}
$$

avec

$e_{\text {air }}$ : émissivité de l'air (un des trois paramètres possible de calage, pris à 
sa valeur standard de 0,8 dans cette étude);

$\sigma:$ constante de Stefan-Boltzman, $\sigma=5,67.10^{-8} \mathrm{Wm}^{-2} \mathrm{~K}^{-4}$;

$\mathrm{T}_{\text {air }}$ : mesure de température d'air à la station météorologique considérée $\left({ }^{\circ} \mathrm{C}\right)$;

$\mathrm{K}$ : coefficient dépendant normalement du type de nuage mais pris classiquement à 0,2.

RE est calculé par l'équation :

$$
\mathrm{RE}=\mathrm{e}_{\text {eau }} \sigma\left(\mathrm{T}_{\text {eau }}+273,15\right)^{4}
$$

avec

$\mathrm{T}_{\text {eau }}$ : température de l'eau $\left({ }^{\circ} \mathrm{C}\right)$ calculée par CALNAT;

$\mathrm{e}_{\text {eau }}$ : émissivité de l'eau prise constante à 0,97.

CV est calculé par l'équation

$$
\mathrm{CV}=\rho_{\text {air }} \mathrm{Cp}_{\text {air }}(\mathrm{a}+\mathrm{bV})\left(\mathrm{T}_{\text {eau }}-\mathrm{T}_{\text {air }}\right)
$$

avec

$\rho_{\text {air }}$ : masse volumique de l'air $\left(\mathrm{kg} / \mathrm{m}^{3}\right)$, donnée par la formule : $\rho_{\text {air }}=$ $\frac{100 \times P_{\text {atm }}}{\left(T_{\text {air }}+273,15\right) \times 287}$ avec $P_{\text {atm }}$ pression atmosphérique ( $\mathrm{hPa}$ ) à la station météorologique considérée;
$C p_{a i r}$ : chaleur massique de l'air $\left(\mathrm{J} . \mathrm{kg}^{-1} \cdot \mathrm{K}^{-1}\right)$;

$\mathrm{V}$ : vitesse du vent à 10 mètres à la station météorologique $(\mathrm{m} / \mathrm{s})$;

$a, b$ : coefficients de la fonction de vent qui sont des paramètres de CALNAT et sont pris à leur valeur stan$\operatorname{dard}^{(14)}$ de 0,002 et 0,0012 dans cette étude.

CE est calculé par l'équation :

$$
C E=L \rho_{\text {air }}(a+b V)\left(H^{s a t}-H u\right)
$$

avec

$\mathrm{L}$ : chaleur latente d'évaporation $(\mathrm{J} / \mathrm{kg})$, donnée par l'équation : $L=$ $2500900-2365 \times \mathrm{T}_{\text {eau }}$;

$\mathrm{Hu}$ : humidité spécifique de l'air $(\mathrm{kg} /$ $\mathrm{kg}$ ), donnée par : $H u=\frac{0,622 \times P_{\text {vap }}}{P_{\text {atm }}-0,378 \times P_{\text {vap }}}$ avec $P_{\text {vap }}$ pression partielle de vapeur d'eau de l'air ( $\mathrm{hPa}$ ) fournie par la station météorologique;

$\mathrm{H}^{\text {sat }}$ : humidité spécifique de l'air saturé à la température de surface de l'eau $\mathrm{T}_{\text {eau }}(\mathrm{kg} / \mathrm{kg})$, donnée par $\mathrm{H}^{\text {sat }}=\frac{0,622 \times \mathrm{P}_{\text {vap }}^{\text {sat }}}{\mathrm{P}_{\mathrm{atm}}-0,378 \times \mathrm{P}_{\text {vap }}^{\text {sat }}}$ avec $\mathrm{P}_{\text {vap }}^{\text {sat }}$ pression partielle de vapeur d'eau à saturation $(\mathrm{hPa})$ calculée au moyen de la formule suivante : $\mathrm{P}_{\mathrm{vap}}^{\mathrm{sat}}=$ $6,11 \exp \left(\frac{17,27 \times T_{\text {eau }}}{T_{\text {eau }}+237,3}\right)$.

(14) On peut trouver des recommandations de 0,0025 et 0,0015 pour a et b dans des études EDF des années 1980. L'examen du code de calcul alors utilisé a montré que ces valeurs notaient en fait les produits $\rho_{\text {air }}$ et $\rho_{\text {air }}$ b avec $\rho_{\text {air }}$ en $\mathrm{kg} / \mathrm{m}^{3}$. Si bien que les vraies valeurs de a et $b$ issues de ces recommandations sont en fait très proches de celles considérées dans notre étude. 


\section{Annexe 2 : Description du test statistique appliqué à l'analyse de tendance de trois modèles de température d'eau (CALNAT, COURNAT, modèle de régression multilinéaire) et de données hydrologiques et météorologiques}

Citation de la thèse de $B$. Renard (2006), page $97^{(15)}$.

Ce test est basé sur le modèle paramétrique suivant :

$$
X_{t}=\alpha+\beta t+\xi_{t}
$$

où les $X_{t}$ est l'ensemble des $\mathrm{n}$ valeurs à disposition pour l'ajustement de la tendance et les erreurs $\xi_{t}$ suivent une loi normale centrée de variance $\sigma^{2}$. Par la méthode des moindres carrés, les paramètres sont estimés de la manière suivante :

$$
\hat{\beta}=\frac{\sum_{t=1}^{n}(t-\bar{t}) X_{t}}{\sum_{t=1}^{n}(t-\bar{t})^{2}}, \hat{\alpha}=\bar{X}-\hat{\beta} \bar{t} .
$$

La variance de l'estimateur de tendance peut être estimée par :

$$
\operatorname{var}(\hat{\beta})=\frac{12 \sum_{t=1}^{n}\left(X_{t}-\hat{\alpha}-\hat{\beta} t\right)^{2}}{(n-2) n\left(n^{2}-1\right)} .
$$

Le test de la régression linéaire consiste alors à vérifier le seuil de signification (significativité) de cette tendance, c'est-à-dire à tester que l'estimateur du coefficient directeur $\beta$ est suffisamment différent de zéro. Pour cela, on compare la statistique de test suivante aux quantiles de la loi de Student à $n-2$ degré de liberté :

$$
Z=\frac{\hat{\beta}}{\sqrt{\operatorname{var}(\hat{\beta})}} .
$$

II s'agit d'un test paramétrique, supposant la normalité des données, et spécialement conçu pour détecter une tendance linéaire. Ce test est cependant réputé robuste à une distribution qui serait éventuellement non gaussienne.
(15) Le calcul de l'intervalle de confiance est fait en considérant une distribution normale de la tendance, de moyenne $\hat{\beta}$ et de variance $\operatorname{var}(\hat{\beta})$. Sur les figures 5 et 6, IC $B_{\text {inf }} 90 \%$ et IC $B_{\text {sup }}$ $90 \%$ signifient borne inférieure et supérieure de l'intervalle de confiance à $90 \%$. 